\title{
Electroweak Baryogenesis in Two Higgs Doublet Models and $B$ meson anomalies
}

\author{
James M. Cline* \\ Department of Physics, McGill University, Montreal, Quebec H3A 2T8, Canada \\ Kimmo Kainulainen \\ Department of Physics, P.O.Box 35 (YFL), \\ FIN-40014 University of Jyväskylä, Finland and \\ Helsinki Institute of Physics, P.O. Box 64, \\ FIN-00014 University of Helsinki, Finland \\ Michael Trott \\ Perimeter Institute for Theoretical Physics, Waterloo, ON N2J-2W9, Canada
}

(Dated: November 18, 2011)

\begin{abstract}
Motivated by $3.9 \sigma$ evidence of a CP-violating phase beyond the standard model in the like-sign dimuon asymmetry reported by $\mathrm{D} \emptyset$, we examine the potential for two Higgs doublet models (2HDMs) to achieve successful electroweak baryogenesis (EWBG) while explaining the dimuon anomaly. Our emphasis is on the minimal flavour violating 2HDM, but our numerical scans of model parameter space include type I and type II models as special cases. We incorporate relevant particle physics constraints, including electroweak precision data, $b \rightarrow s \gamma$, the neutron electric dipole moment, $R_{b}$, and perturbative coupling bounds to constrain the model. Surprisingly, we find that a large enough baryon asymmetry is only consistently achieved in a small subset of parameter space in $2 \mathrm{HDMs}$, regardless of trying to simultaneously account for any $B$ physics anomaly. There is some tension between simultaneous explanation of the dimuon anomaly and baryogenesis, but using a Markov chain Monte Carlo we find several models within $1 \sigma$ of the central values. We point out shortcomings with previous studies that reached different conclusions. The restricted parameter space that allows for EWBG makes this scenario highly predictive for collider searches. We discuss the most promising signatures to pursue at the LHC for EWBG-compatible models.
\end{abstract}




\section{Contents}

\section{Introduction}

II. Model
A. $\mathrm{CP}$ violation
B. Relations to other Two Higgs Doublet models
C. Fitting to the $\mathrm{D} \varnothing$ and other $B$ sector anomalies
D. Higher order terms in the MFV expansion

\section{Phenomenological Constraints}

A. Collider Mass bounds

14

B. Consistency Conditions on the Potential

15

C. Phenomenological constraints on the loop corrections due to charged scalars

1. Neutron EDM

2. $Z \rightarrow b \bar{b}$ constraints

3. Constraints on $\zeta_{t}^{\prime \pm}$ through $b \rightarrow s \gamma$

D. Electroweak precision data

\section{Finite temperature effective potential}
A. Counterterms and Goldstone bosons
B. Thermal corrections

21

22

\section{Electroweak phase transition}
A. Finding $T_{c}$
B. Search strategy
C. Results

\section{Baryogenesis}

A. Bubble wall path and the CP-violating phase of the top quark

\footnotetext{
*Electronic address:jcline@ hep.physics.mcgill.ca

$\dagger$ Electronic address kimmo.kainulainen@jyu.fi

${ }^{\ddagger}$ Electronic address mtrott@ perimeterinstitute.ca
} 
2. Spatially varying phase of $m_{t}$

31

3. Numerical solution for bubble wall profiles

4. Comparison with previous results

B. Transport equations and source term

1. The source term 36

2. Transport equations 37

C. Baryon asymmetry results 38

VII. Experimental testability 41

A. Confirmation/refutation of the dimuon anomaly 42

B. Collider prospects for 2HDMs with successful EWBG 44

1. Tevatron Physics 44

2. LHC Physics 45

VIII. Discussion and conclusions

Acknowledgments

A. Counterterms

B. Field-dependent masses

C. Beta functions

References

\section{INTRODUCTION}

An experimental hint for beyond the Standard Model (SM) CP violation was recently reported by the $\mathrm{D} \varnothing$ collaboration [1, 2]. The like-sign dimuon asymmetry in semileptonic $B$ decay has been observed to deviate from the SM prediction, initially with a statistical significance of $3.2 \sigma$. As this paper approached completion the statistical significance of this anomaly was reported to increase [1] to $3.9 \sigma$, while the central value shifted within the error band of the previous measure- 
ment. ${ }^{1}$ This has motivated us to revisit electroweak baryogenesis (EWBG) in two Higgs doublet models. ${ }^{2}$ This measurement could indicate a new CP-violating phase contributing to the mixing of neutral $B$ mesons or it could be a statistical fluctuation. Interpreting this observation as a sign of electroweak scale new physics is supported by a pattern of deviations measured from the SM in the $B$ sector. $^{3}$ This is also interesting as the measured CP violation of the SM is well known to be insufficient to generate the baryon asymmetry of the universe in electroweak baryogenesis (EWGB) scenarios. In this paper, we systematically reexamine the possibility of EWGB in two Higgs doublet models with new sources of $\mathrm{CP}$ violation in light of this experimental pattern.

In any model with multiple scalar doublets, some symmetry (usually approximate) must be invoked to suppress flavour changing neutral currents (FCNC's). Frequently, treatments of multiscalar doublet models impose ad-hoc discrete symmetries on the couplings of quarks and leptons to the two doublets, following Weinberg and Glashow [10]. In this work, we focus on the framework of Minimal Flavour Violation (MFV) [11-13]. This approach allows a new physics sector with multiple scalar doublets that is naturally consistent with flavour constraints without such discrete symmetries. For the purposes of testing electroweak baryogenesis, the MFV 2HDM is sufficiently general that previously considered "type I" and "type II" models can be thought of as special cases of the MFV Yukawa couplings. Therefore our analysis applies to a broad class of 2HDMs, even though our emphasis is on the MFV framework. Ref. [14] has recently considered EWBG in a 2HDM which does not fit into any of these categories, but instead has couplings to the extra singlet that are engineered to reproduce the $\mathrm{D} \phi$ anomaly while still respecting constraints on FCNCs.

Electroweak baryogenesis in 2HDMs has been discussed in a number of previous papers [1520] and is the prototypical model for EWBG, where the dynamical source of CP violation is the simplest: it is the spatially varying phase $\theta(z)$ of the top quark mass. ${ }^{4}$ With the exception of ref.

\footnotetext{
${ }^{1}$ After this paper appeared, two new measurements were published. A new result from $D \emptyset[3]$ is still consistent with the dimuon anomaly, while a new result from $\mathrm{LHC}_{\mathrm{b}}$ is in tension with the dimuon anomaly. We discuss these results in more detail in Section VII

${ }^{2}$ Only one linear combination of fields plays the role of the Higgs boson, so these should more properly be called "two scalar doublet models," but here we adhere to the customary convention.

${ }^{3}$ The measurements of $B_{s} \rightarrow J / \psi \phi$ and $B^{-} \rightarrow \tau \nu$ also hint at the possibility of a new phase in $B_{s, d}$ mixing (for a recent global fit and discussion see [4]). These deviations have the correct correlations to be part of a consistent pattern pointing to a new CP-violating phase. The deviation of $B^{-} \rightarrow \tau \nu$ has the most statistical significance-it is a $2.6 \sigma$ deviation between the SM expectation [4] to the averaged measurements of $B^{-} \rightarrow \tau \nu$ performed at Belle and Babar [5-8]. See [9] for a recent discussion of $B^{-} \rightarrow \tau \nu$ in this model framework.

${ }^{4}$ Even though the MSSM has two Higgs doublets, it provides no such phase for $m_{t}$ at tree level because of the restricted form of the couplings in the Higgs potential.
} 
[20], these papers were written before there was a consensus on the proper way to compute the source term that appears in the Boltzmann equations needed to solve for the baryon asymmetry. Thus ref. [20] gives the most complete treatment to date; yet it leaves much room for the improvements that we undertake in the present work. We point out a heretofore unnoticed effect that systematically suppresses the magnitude of the phase $\theta(z)$, making it challenging to get a large enough baryon asymmetry. We note that actually solving for the bubble wall profile rather than merely parametrizing it, as was done in [20], typically leads to smaller results for the baryon asymmetry.

We also find that the particle physics constraints imposed here, but not in previous works, significantly reduce the parameter space that can succeed for baryogenesis. Using Monte Carlo methods we scan over the full allowed parameter space of the model consistent with these constraints, rather than restricting it in an ad-hoc manner, as has been done in previous studies.

The present work should be regarded first as a fairly general analysis of EWBG in 2HDMs, since as we will argue, the MFV framework is broad enough to also encompass some popular 2HDMs (Type I and Type II) that have been considered before. Beyond this, we also consider whether it is possible to simultaneously account for EWBG and the level of new CP violation in the $B$ sector suggested by the $\mathrm{D} \emptyset$ anomaly. It will be shown that the two effects are largely uncorrelated, due to the fact that we do not find significant EWBG associated with new $\mathrm{CP}$ violation coupling to $b$ quarks; rather it must exsit in the top quark Yukawa coupling for this purpose. In fact, we will find some tension between simultaneously explaining the $\mathrm{D} \varnothing$ dimuon observation and getting sufficient baryogenesis.

The paper is organized as follows. In section $\Pi$ we describe the 2HDM model with MFV and fitting to the dimuon anomaly. Section [II]summarizes the various phenomenological and theoretical consistency constraints that we impose when scanning the parameter space of the model. In section $[\nabla$ we construct the one-loop finite-temperature effective potential that is used to determine properties of the electroweak phase transition. Section $\nabla$ describes the results of scanning over the model parameter space, using a Markov chain Monte Carlo (MCMC), to find models in which the electroweak phase transition (EWPT) is strong enough for EWBG. In section VI] we study the baryon asymmetry generated by these models, finding that it tends to be somewhat below the observed value when the dimuon anomaly is reproduced, although exceptions can be found. Even when the dimuon anomaly is neglected, only relatively rare examples exist that can produce the observed baryon asymmetry. We then discuss in section VIIthe experimental prospects at LHC for 
confirming the dimuon anomaly and the MFV two Higgs doublet model, assuming that successful EWBG occurs. Conclusions are given in section VIII.

\section{MODEL}

We begin with some remarks motivating the choice of MFV as our principle for suppressing FCNCs. The mass scale suppressing the operators of interest enhancing $B_{s, d}$ mixing (for perturbative couplings) is a few hundred GeV [21, 22]. Models that seek to explain the D $\varnothing$ measurement with such a mass scale are strongly constrained by flavour changing measurements that agree well with the SM. New physics (NP) models with MFV are naturally consistent with such flavour constraints (in the Glashow-Weinberg sense [10]) as the quark flavour group $\mathrm{G}_{\mathrm{F}}=\mathrm{SU}(3)_{\mathrm{U}_{\mathrm{R}}} \times \mathrm{SU}(3)_{\mathrm{D}_{\mathrm{R}}} \times \mathrm{SU}(3)_{\mathrm{Q}_{\mathrm{L}}}$ is only broken by the SM quark Yukawa couplings $g_{U / D}$ defined as

$$
\mathcal{L}_{Y}=g_{U}{ }_{j}^{i} \bar{u}_{i R} H^{T} \in Q_{L}^{j}-g_{D}{ }_{j}^{i} \bar{d}_{i R} H^{\dagger} Q_{L}^{j}+\text { h.c.. }
$$

where

$$
\epsilon=\left(\begin{array}{cc}
0 & 1 \\
-1 & 0
\end{array}\right)
$$

MFV models do allow new CP-violating phases that can lead to the D $\varnothing$ signal [23-25] and can possibly supply the required extra CP violation for EWGB. They provide an interesting framework for examining the relation between these experimental anomalies and the possibility of EWGB in SM extensions. It is also interesting to assess the feasibility of EWBG in multi-scalar doublet models with MFV, regardless of whether the dimuon anomaly is confirmed by future experiments.

We denote by $\mathrm{H}$ the doublet that gets a vacuum expectation value (by definition the Higgs) and by $\mathrm{S}$ the doublet that does not. One can always rotate to a field basis where this is the case in the models we consider. Our discussion of the model will largely follow [25]. The Lagrangian in the Yukawa sector is

$$
\mathcal{L}_{Y}=g_{U j}^{i} \bar{u}_{i R} H^{T} \epsilon Q_{L}^{j}-g_{D}{ }^{i}{ }_{j} \bar{d}_{i R} H^{\dagger} Q_{L}^{j}+Y_{U j}^{i} \bar{u}_{i R} S^{T} \epsilon Q_{L}^{j}-Y_{U j}^{i} \bar{d}_{i R} S^{\dagger} Q_{L}^{j}+\text { h.c. }
$$

where flavour indices $i, j$ are shown and summed over and color and $\mathrm{SU}(2)_{\mathrm{L}}$ indices have been suppressed. MFV asserts that any $\mathrm{NP}$ also has $\mathrm{G}_{\mathrm{F}}$ only broken by insertions proportional to Yukawa matrices, so that $Y_{U}{ }_{i}^{j}, Y_{D}{ }_{i}^{j}$ are proportional to $g_{U}{ }_{i}^{j}, g_{D}^{j}{ }_{i}$. One can construct allowed NP 
terms by treating the Yukawa matrices as spurion fields that transform under flavour rotations as

$$
g_{U} \rightarrow V_{U} g_{U} V_{Q}^{\dagger}, \quad g_{D} \rightarrow V_{D} g_{D} V_{Q}^{\dagger},
$$

where $V_{U}$ is an element of $\mathrm{SU}(3)_{\mathrm{U}_{\mathrm{R}}}, V_{D}$ is an element of $\mathrm{SU}(3)_{\mathrm{D}_{\mathrm{R}}}$, and $V_{Q}$ is an element of $\mathrm{SU}(3)_{\mathrm{Q}_{\mathrm{L}}}$, i.e., the Yukawa matrices transform as $g_{U} \sim(\mathbf{3}, \mathbf{1}, \overline{\mathbf{3}})$ and $g_{D} \sim(\mathbf{1}, \mathbf{3}, \overline{\mathbf{3}})$ under $\mathrm{G}_{\mathrm{F}}$. MFV can be formulated up to linear order in top Yukawa insertions, or extended to a nonlinear representation of the symmetry [26, 27]. For enhanced CP violation in $B_{q}$ mixing we are interested in (at least) the second order terms in the expansion of the top Yukawa in MFV. It is sufficient in our initial discussion to only expand to next order in insertions of $g_{U}$ so that

$$
\begin{aligned}
Y_{U}{ }_{i}^{j} & =\eta_{U} g_{U i}^{j}+\eta_{U}^{\prime} g_{U k}^{j}\left[\left(g_{U}^{\dagger}\right)_{l}^{k}\left(g_{U}\right)_{i}^{l}\right]+\cdots, \\
Y_{D}{ }_{i}^{j} & =\eta_{D} g_{D i}^{j}+\eta_{D}^{\prime} g_{D}^{j}{ }_{k}\left[\left(g_{U}^{\dagger}\right)_{l}^{k}\left(g_{U}\right)_{i}^{l}\right]+\cdots .
\end{aligned}
$$

We decompose the second scalar doublet as $S^{T}=\left(S^{+}, S^{0}\right)$, where $S^{0}=\left(s_{R}+i s_{I}\right) / \sqrt{2}$. The scalar potential is

$$
\begin{aligned}
V & =\frac{\lambda}{4}\left(H^{\dagger i} H_{i}-\frac{v^{2}}{2}\right)^{2}+m_{1}^{2}\left(S^{\dagger i} S_{i}\right)+\left(m_{2}^{2} H^{\dagger i} S_{i}+\text { h.c. }\right) \\
& +\lambda_{1}\left(H^{\dagger i} H_{i}\right)\left(S^{\dagger j} S_{j}\right),+\lambda_{2}\left(H^{\dagger i} H_{j}\right)\left(S^{\dagger j} S_{i}\right)+\left[\lambda_{3} H^{\dagger i} H^{\dagger j} S_{i} S_{j}+\text { h.c. }\right] \\
& +\left[\lambda_{4} H^{\dagger i} S^{\dagger j} S_{i} S_{j}+\lambda_{5} S^{\dagger i} H^{\dagger j} H_{i} H_{j}+\text { h.c. }\right]+\lambda_{6}\left(S^{\dagger i} S_{i}\right)^{2}
\end{aligned}
$$

where $i, j$ are $\mathrm{SU}(2)$ indices. Here $v \simeq 246 \mathrm{GeV}$ is the vacuum expectation value (VEV) of the Higgs field. Since we adopt the convention that the doublet $S$ does not get a VEV the parameters $m_{2}^{2}$ and $\lambda_{5}$ are related by,

$$
m_{2}^{2}+\lambda_{5}^{\star} \frac{v^{2}}{2}=0
$$

The spectrum of neutral real scalar fields consists of the Higgs scalar $h=\sqrt{2} \Re\left(H^{0}\right)$ another scalar field $s_{R} \equiv \sqrt{2} \Re\left(S^{0}\right)$ and a pseudoscalar $s_{I} \equiv \sqrt{2} \Im\left(S^{0}\right)$. However, these are not mass eigenstates; in the $\left(h, s_{R}, s_{I}\right)$ basis the neutral mass squared matrix $\mathcal{M}^{2}$ is

$$
\mathcal{M}^{2}=\left(\begin{array}{ccc}
m_{h}^{2} & \lambda_{5}^{R} v^{2} & \lambda_{5}^{I} v^{2} \\
\lambda_{5}^{R} v^{2} & m_{H}^{2} & 0 \\
\lambda_{5}^{I} v^{2} & 0 & m_{A}^{2}
\end{array}\right)
$$

where ${ }^{5}$

$$
m_{h}^{2} \equiv \lambda v^{2} / 2, \quad m_{H}^{2} \equiv m_{S}^{2}+\lambda_{3} v^{2} \quad \text { and } \quad m_{A}^{2} \equiv m_{S}^{2}-\lambda_{3} v^{2}
$$

\footnotetext{
${ }^{5}$ We make $\lambda_{3}$ real by a phase rotation of $S$ with respect $H$. We also define $\lambda_{4}=\lambda_{4}^{R}+i \lambda_{4}^{I}$ and $\lambda_{5}=\lambda_{5}^{R}+i \lambda_{5}^{I}$
} 
with $m_{S}^{2} \equiv m_{1}^{2}+\left(\lambda_{1}+\lambda_{2}\right) v^{2} / 2$. Note that $m_{H}, m_{A}$ is associated with $s_{R}, s_{I}$. The mass eigenstate field basis is denoted as $h^{\prime}, s_{R}^{\prime}, s_{I}^{\prime}$ and can be expanded in terms of the original field basis as

$$
h^{\prime}=h-\epsilon_{S_{R}} s_{R}-\epsilon_{S_{I}} s_{I}, \quad s_{R}^{\prime}=s_{R}+\epsilon_{S_{R}} h, \quad \text { and } \quad s_{I}^{\prime}=s_{I}+\epsilon_{S_{I}} h
$$

where we defined the expansion parameters

$$
\epsilon_{S_{R}} \equiv \frac{v^{2} \lambda_{5}^{R}}{m_{H}^{2}-m_{h}^{2}} \quad \text { and } \quad \epsilon_{S_{I}} \equiv \frac{v^{2} \lambda_{5}^{I}}{m_{A}^{2}-m_{h}^{2}} .
$$

The general Wilson coefficient for the relevant operator in the effective Hamiltonian $\mathcal{H}_{q}^{\mathrm{NP}} \simeq$ $\left(V_{t q}^{\star} V_{t b}\right)^{2} C^{\mathrm{NP}}\left(m_{t}\right) \bar{b}_{R}^{\alpha} q_{L}^{\alpha} \bar{b}_{R}^{\beta} q_{L}^{\beta}$ for perturbative $\lambda_{5}^{R, I} \ll 1$ to leading nontrivial order in the MFV expansion is

$$
C^{\mathrm{NP}}\left(m_{t}\right)=\left(\eta_{D}^{\prime}\right)^{2} y_{t}^{4}\left\{\frac{\left(\lambda_{3}+\epsilon_{S_{R}} \lambda_{5}^{R}-\epsilon_{S_{I}} \lambda_{5}^{I}\right) m_{b}^{2}}{m_{H}^{2} m_{A}^{2}}+\frac{\left(\epsilon_{S_{R}}^{2}+\epsilon_{S_{I}}^{2}\right) m_{b}^{2}}{2 v^{2} m_{h}^{2}}\right\},
$$

where $y_{t} \equiv \sqrt{2} m_{t} / v$. In the above equation, the bottom quark mass $m_{b} \simeq 2.93 \mathrm{GeV}$ is evaluated at the top quark mass scale. Here the Wilson coefficient was obtained under the assumptions $m_{H}, m_{A}>m_{h}, \epsilon_{S_{R}}, \epsilon_{S_{I}} \ll 1$. In our convention the CKM factors are pulled out of the Wilson coefficient and appear explicitly in the effective Hamiltonian. Note that the Wilson coefficient is proportional to $\left(\eta_{D}^{\prime}\right)^{2}$, not $\left|\eta_{D}^{\prime}\right|^{2}$. In general this parameter is complex, and the dependence on $\left(\eta_{D}^{\prime}\right)^{2}$ introduces an extra phase into the mixing of $B_{s}$ mesons through this Wilson coefficient.

For EWBG, we will make use of the different but analogous phase that comes from the MFV expansion of the Yukawa couplings in the top sector. However to explain both of these anomalies simultaneously a number of phenomenological constraints common to the physics of both effects must be accommodated. For example, the mass scales of the new scalar states are relevant both for achieving successful baryogenesis and for explaining the dimuon anomaly.

\section{A. CP violation}

Since new CP violation is a central ingredient to this work, let us summarize the new CPviolating phases in our model. The new Yukawa couplings to the extra Higgs field $S$ are beyond the standard model, and their phases cannot be removed by field redefinitions, which have already been used to push the phases in the SM-like Yukawa couplings (to the Higgs $H$ ) into the CKM matrix. For our purposes, the relevant new couplings are to the heaviest quarks, $t$ and $b$, the first of which is important for baryogenesis and the second for the dimuon anomaly. 
Beyond these two new phases, the Higgs potential has four complex couplings, $m_{2}^{2}, \lambda_{3}, \lambda_{4}$ and $\lambda_{5}$. Because of our convention that $S$ does not get a VEV at zero temperature, eq. (7), $m_{2}^{2}$ and $\lambda_{5}$ are linearly related, removing one of these phases. Moreover we can do a phase rotation on $S$ (relative to $H$ ) to remove an additional phase; we choose to make $\lambda_{3}$ real. Thus there are two unremovable phases in the Higgs potential, in addition to the two new relevant phases in the $S$ Yukawa couplings. We will show (see sect. $\left(\mathrm{VB}\right.$ ) that nonzero $\lambda_{5}$ suppresses the strength of the electroweak phase transition, so its phase will not play an important role for us. The effect of the phase of $\lambda_{4}$ is to allow for $S$ to be complex inside the bubble walls of the EWPT, inducing a phase difference $\varphi_{s h}$ between the neutral components of $S$ and $H$. It is the sum of $\varphi_{s h}$ and the phase of the new top quark Yukawa coupling, denoted by $\varphi_{\eta}$, that will appear in the spatially-dependent CP-violating phase in the bubble wall, $\theta(z)$; see eq. (61).

\section{B. Relations to other Two Higgs Doublet models}

We will show that only relatively rare examples exist leading to a large enough baryon asymmetry in this model. We believe that paucity of models producing a large enough baryon asymmetry also holds for traditional type I or II two-Higgs doublet models as we now explain. Consider a type I or II two-Higgs doublet model where the matrix Yukawa couplings to $H_{1}$ and $H_{2}$ satisfy

$$
\begin{aligned}
& g_{U}^{2}=g_{D}^{2}=0, \quad \text { type I, } \\
& g_{U}^{2}=g_{D}^{1}=0, \text { type II, }
\end{aligned}
$$

and $g_{U, D}^{i}$ denotes the diagonal Yukawa couplings of the scalar fields to up or down quarks respectively. Each of these Yukawa couplings is multiplied by $\eta_{U}^{i}$ and $\eta_{D}^{i}$. In this notation, both fields generically get VEV's in the ratio $\left\langle H_{2}\right\rangle /\left\langle H_{1}\right\rangle=\tan \beta$. The form of the couplings (12) can be insured by $Z_{2}$ symmetries, but in order to get a CP-violating phase in the Higgs sector for EWBG, these symmetries need to be broken; no unbroken symmetry distinguishes the scalar fields.

If the symmetry were exact, then its action on the fields could be obscured by rotating to a basis in the $H_{1}, H_{2}$ space. But since the symmetry is broken, nothing inhibits us from redefining fields to go to a new basis where $H_{1}$ is along the symmetry breaking direction and $H_{2}$ gets no VEV. By transforming the Yukawa couplings accordingly, we immediately find that the broken type I model corresponds to the general MFV model with the condition $\eta_{U / D}^{1}=-\eta_{U / D}^{2} \cot \beta$. Similarly, the broken type II model corresponds to the condition $\eta_{U}^{1}=-\eta_{U}^{2} \cot \beta$ and $\eta_{D}^{2}=-\eta_{D}^{1} \cot \beta$. We have set all other parameters $\eta_{U}^{\prime}, \eta_{U}^{\prime \prime}, \eta_{D}^{\prime}, \eta_{D}^{\prime \prime}+\cdots$ for both scalar fields to zero in this discussion 
for simplicity. Our MCMC exploration of the parameter space would tend toward these relations between the couplings if they were favorable toward EWBG. The fact that they do not shows that the flavour structure of this model is not responsible for the difficulty of achieving EWBG in our analysis.

This demonstrates that the MFV 2HDM is a general framework that includes type I, II models (in which the discrete symmetry is softly broken, as is always required to get EWBG) where both scalar fields couple to the quarks as special cases. For the purposes of only achieving EWBG, in fact there is no interesting distinction between the models, since as we will confirm the top quark Yukawa couplings by themselves give the dominant contribution to the baryon asymmetry. Thus, if setting aside the question of $B$ physics, we can set $\eta_{D}^{i}$ to zero. Then the relations between $\eta_{U}^{i}$ to reproduce type I or II Yukawa structure can always be satisfied for some choice of $\tan \beta$. To the extent that the top quark source dominates EWBG, there is no significant difference between type I, II or MVF models.

\section{Fitting to the $\mathrm{D} \emptyset$ and other $B$ sector anomalies}

When attempting to link EWBG to the recent dimuon anomaly, we use the fit of [4] to determine the new contribution to $B_{q}-\bar{B}_{q}$ mixing (here $q=s, d$ ). The $\mathrm{D} \varnothing$ result that deviates from the SM prediction at $3.2 \sigma\left(a_{S L}^{b}\right)$ and the SM prediction $\left(A_{S L}^{b}\right)$ are given by

$$
\begin{aligned}
a_{S L}^{b} & =\frac{N_{b}^{++}-N_{b}^{--}}{N_{b}^{++}+N_{b}^{--}}, \\
& =-(9.57 \pm 2.51 \pm 1.46) \times 10^{-3}, \\
A_{S L}^{b} & =\left(-3.10_{-0.98}^{+0.83}\right) \times 10^{-4} .
\end{aligned}
$$

The number of $X b \bar{b} \rightarrow \mu^{+} \mu^{+} Y$ events is given by $N_{b}^{++}$for example. The quoted $a_{S L}^{b}$ is a combination of the asymmetry in each $B_{q}$, denoted $a_{S L}^{b q}$. Each of these contributions to $a_{S L}^{b}$ can be expressed in terms of the mass and width differences $\left(M_{12}, \Gamma_{12}\right)$ of the $B_{q}$ meson eigenstates and the $\mathrm{CP}$ phase difference between these quantities $\phi_{q}$ as

$$
a_{S L}^{b q}=\frac{\left|\Gamma_{12}^{q}\right|}{\left|M_{12}^{q}\right|} \sin \phi_{q} .
$$

The model we discuss involves a NP contribution that includes a new CP-violating phase to $M_{12}^{q}$. It also allows other new CP-violating phases, all of which are interesting for our study, due to their 
potential to drive EWBG. The effect of this model on $B_{s}$ and $B_{d}$ mass mixing is encoded in two real parameters, $h_{q}>0$ and $\sigma_{q}$, by writing

$$
M_{12}^{q}=\left(M_{12}^{q}\right)^{\mathrm{SM}}+\left(M_{12}^{q}\right)^{\mathrm{NP}}, \quad\left(M_{12}^{q}\right)^{\mathrm{NP}}=\left(M_{12}^{q}\right)^{\mathrm{SM}} h_{q} e^{2 i \sigma_{q}} .
$$

Frequently in NP models that obey MFV one has a flavour universal phase, so that $h_{s}=h_{d}$ and $\sigma_{s}=\sigma_{d}$, and the best fit values are $h_{q}=0.255$ and $2 \sigma_{q}=180^{\circ}+63.4^{\circ}$. This scenario is argued to be a better fit to the current data then the SM in [4], which is disfavoured with a p-value of $3.1 \sigma$. A recent update of this measurement by $\mathrm{D} \phi$ with $9 \mathrm{fb}^{-1}$ of data finds that the significance of the deviation has increased to $3.9 \sigma$. We continue to utilize the global fit results when attempting to accommodate the dimuon anomaly and EWGB, as a new global best fit value is not available. The updated result is within the error band of the previous measurement that is incorporated into the global fit, so we expect our conclusions to hold for an update to the global fit incorporating the more statistically significant deviation.

We treat perturbative QCD in the leading logarithmic approximation and evaluate the needed matrix elements of four quark operators using the vacuum insertion approximation at the $b$ quark mass scale. The effective Hamiltonian is $\mathcal{H}_{q}^{\mathrm{NP}} \simeq\left(V_{t q}^{\star} V_{t b}\right)^{2} C^{\mathrm{NP}}\left(m_{t}\right) \bar{b}_{R}^{\alpha} q_{L}^{\alpha} \bar{b}_{R}^{\beta} q_{L}^{\beta}$ (see Eqn. (11)) where $\alpha, \beta$ are colour indices; one finds

$$
\left(M_{12}^{q}\right)^{\mathrm{NP}} \simeq\left(V_{t q}^{\star} V_{t b}\right)^{2} C^{\mathrm{NP}}\left(m_{t}\right)\left(-\frac{5}{24}\right) \eta^{\prime} f_{B_{q}}^{2} m_{B_{q}}
$$

with the Wilson coefficient defined as above. Using the results of [25] we have

$$
h_{q} e^{2 i \sigma_{q}} \simeq-\frac{5}{8}\left(\frac{C^{\mathrm{NP}}\left(m_{t}\right)}{C^{\mathrm{SM}}\left(m_{t}\right)}\right) \frac{\eta^{\prime}}{\eta} .
$$

where $\eta \simeq 0.84$ is a QCD correction factor, and $\eta^{\prime} \simeq 1.45$. The SM contribution is

$$
C^{\mathrm{SM}}\left(m_{t}\right)=\frac{G_{F}^{2}}{4 \pi^{2}} M_{W}^{2} S\left(\frac{m_{t}^{2}}{M_{W}^{2}}\right) .
$$

where $S\left(m_{t}^{2} / M_{W}^{2}\right) \simeq 2.35$ [28]. Using these results we can scan over the allowed parameter space in the potential parameters $\lambda_{i}$, couplings $\eta_{i}$, and the masses of the new scalars, to search for examples consistent with successful EWBG while fitting the dimuon anomaly.

\section{Higher order terms in the MFV expansion}

Flavor breaking in MFV is based on an expansion in the insertions of the spurions $Y_{U}^{\dagger} Y_{U}$ and $Y_{D}^{\dagger} Y_{D}$. These spurions can be inserted between any contraction of the $Q_{L}$ flavour indices. 
Each term in the expansion where a spurion is inserted comes with an unknown parameter $\eta_{U}, \eta_{U}^{\prime}, \eta_{U}^{\prime \prime} \cdots$. If these parameters are $O(1)$, it is not appropriate to expand to only leading order when considering the effects of the top Yukawa. ${ }^{6}$ Formally, one can treat these higher order terms in the elegant GMFV approach of [27]. In this section, we discus higher order terms in this expansion and their impact on the MFV 2HDM model. We adopt a less elegant, but simpler approach than [27] for our constraints.

We define new couplings, $\zeta_{U}, \zeta_{U}^{\prime}, \zeta_{D}, \zeta_{D}^{\prime}$, that resum parts of the series in the original expansion. The couplings $\zeta_{U}, \zeta_{D}$ are defined as the sum of all terms in the usual MFV expansion that do not involve flavour change, while the couplings $\zeta_{U}^{\prime}, \zeta_{D}^{\prime}$ are defined as the sum of all terms in the expansion in $\eta_{i}\left(\sqrt{2} m_{t} / v\right)^{2}$ that do lead to a particular flavour change. For couplings to neutral scalar fields this $t \rightarrow j$ flavour change is from one charge $+2 / 3$ quark species to another. For couplings to the charged scalar fields, the flavour change is from an up type quark to a down type quark. In each case, in MFV, the transition is accompanied by the appropriate CKM suppression of the standard model. Then for the neutral scalar fields one has for the top couplings

$$
\begin{aligned}
\zeta_{t}^{0} & =y_{t}\left(\eta_{U}+\eta_{U}^{\prime} y_{t}^{2}+\eta_{U}^{\prime \prime} y_{t}^{4}+\cdots\right), \\
\zeta_{t}^{\prime 0} & =\left(V_{b t}^{\star} V_{b j}\right) y_{t}\left(\tilde{\eta}_{U}^{\prime} y_{b}^{2}+\tilde{\eta}_{U}^{\prime \prime} y_{b}^{4}+\cdots\right),
\end{aligned}
$$

where $y_{t, b}=\sqrt{2} m_{t, b} / v$. For flavour change one must insert $g_{D}^{\dagger} g_{D}$ spurions as a correction to the $g_{U}$ coupling as flavour changing effects require the presence of both the up and down Yukawa matrices. These insertions, since they arise from a different spurion insertion, come with different parameters which we denote $\tilde{\eta}_{U}^{\prime}, \tilde{\eta}_{U}^{\prime \prime}$ in the expansion of $\zeta_{t}^{\prime 0}$. There are also $g_{D}^{\dagger} g_{D}$ insertions in the expansion of $\zeta_{t}^{0}$, but we neglect them in this expression. For the charged fields one has for the top couplings generating the $t \rightarrow b$ flavour change

$$
\begin{aligned}
\zeta_{t}^{ \pm} & =0 \\
\zeta_{t}^{\prime \pm} & =\left(V_{b t}\right) y_{t}\left(\eta_{U}+\eta_{U}^{\prime} y_{t}^{2}+\eta_{U}^{\prime \prime} y_{t}^{4}+\cdots\right)
\end{aligned}
$$

There are also $g_{D}^{\dagger} g_{D}$ insertions in the expansion of $\zeta_{t}^{ \pm}$exactly as in the expansion of $\zeta_{t}^{0}$. For the purposes of this discussion we neglect terms subleading in CKM or small Yukawa contributions to the effective couplings. The effective charged coupling multiplying the CKM insertion is the

\footnotetext{
${ }^{6}$ In the discussion above, we have expanded to the leading order required for the $B_{s, d}$ mixing effect that we are interested in, avoiding these complications until this section in for the sake of clarity.
} 
same as the effective coupling for the neutral field when no flavour change occurs. Furthermore $V_{t b} \sim 1$ to a good approximation. This identification allows one to directly relate the requirements of EWGB to the particle physics constraints that we impose. The procedure above can be repeated for the bottom quark couplings by defining $\zeta_{b}^{0}, \zeta_{b}^{\prime 0}, \zeta_{b}^{ \pm}, \zeta_{b}^{\prime \pm}$.

For the dimuon anomaly, we are interested in the effective flavour changing couplings involving the bottom quark for the new neutral scalar fields. We approximated this coupling with only the leading term in the previous section as $\left(V_{t b}^{\star} V_{t s}\right) \eta_{D}^{\prime} y_{b} y_{t}^{2}$. It is explicitly given by

$$
\zeta_{b}^{\prime 0}=\left(V_{t b}^{\star} V_{t s}\right) y_{b}\left(\eta_{D}^{\prime} y_{t}^{2}+\eta_{D}^{\prime \prime} y_{t}^{4}+\cdots\right)
$$

so that the full result for the Wilson coefficient of interest for enhanced $B_{s}$ mixing is given by

$$
C^{\mathrm{NP}}\left(m_{t}\right)=\left[\frac{\zeta_{b}^{\prime 0}}{V_{t b}^{\star} V_{t s} y_{b}}\right]^{2} \tilde{C}
$$

where $\tilde{C}$ is the quantity in curly brackets in Eqn. (11). The expression $\zeta_{b}^{\prime \pm}$ appears in the precision measurement constraint formulae in the next section. When considering this scenario and fitting to the dimuon anomaly we will take

$$
\zeta_{b}^{\prime \pm} /\left(V_{b t}\right)=f \zeta_{b}^{\prime 0} /\left(V_{t b}^{\star} V_{t s}\right)
$$

with $f=O(1)$ as a generic relation between $\zeta_{b}^{\prime 0}$, which controls the strength of the dimuon anomaly, and $\zeta_{b}^{\prime \pm}$ which appears in the neutron EDM and $b \rightarrow s \gamma$ constraints, as explained in section [II]. The motivation for this choice is that these parameters get the same contributions from all the primed terms in the MFV expansion, while $\eta_{D}$ only appears in $\zeta_{b}^{\prime}$. In the absence of finetuning (or some principle provided by the unknown UV completion of our theory), we expect this relation to hold with $f \sim 1$. However if $\eta_{D}$ happens to partially cancel the other contributions to $\zeta_{b}^{\prime} \pm$, or if the addition of all the spurion insertion terms sums to a suppressed value (compared to $\eta_{D}$ ), it is possible to get a relaxation of the loop-generated constraints relative to the $B$-mixing contribution, hence smaller values of $f$. We will explore the dependence on $f$ in the subsequent analysis.

Aside from taking $f \ll 1$, another way of increasing the $B$-mixing contribution without simultaneously increasing the unwanted $b \rightarrow s \gamma$ and EDM contributions is to increase $\left|\lambda_{5}\right|$, which causes mixing between the scalar CP eigenstates. However this is not helpful in the current situation. The problem is that the part of the function $\tilde{C}$ that can be large enough to generate the dimuon anomaly (with smaller $\left.\eta_{D}^{\prime}\right)$ is proportional to $\left(\lambda_{5}^{R, I}\right)^{2}$ and the first order phase transition required 
for EWBG strongly prefers $\left|\lambda_{5}^{R, I}\right| \ll 1$. In physical terms, the mass mixing between $H$ and $S$ that is driven by $\lambda_{5}$ to allow the dimuon anomaly to be fit to (with smaller $\eta_{D}^{\prime}$ ) directly suppresses the first order phase transition required for EWBG. This is discussed in more detail in Section DI] If $\left|\lambda_{5}^{R, I}\right| \ll 1$, the mass and VEV eigenstates of the scalar fields approximately coincide, which makes discovery of the new scalar states challenging above the $t \bar{t}$ threshold as we will discuss in Section VII

\section{PHENOMENOLOGICAL CONSTRAINTS}

In this section we discuss the constraints on the 2HDM model that we impose when searching for parameter space with viable EWBG. The constraints are enumerated in Table I, which specifies the model parameters that are most directly affected by each one for the reader who wishes to skip the details.

\begin{tabular}{|c|c|}
\hline \hline Constraint & Constrained Parameters \\
\hline LEP/Tevatron Direct Search & $m_{1}, \lambda_{1}, \lambda_{2}, \zeta_{t}^{\prime \pm}$ \\
RGE/Landau Pole/Unitarity & $\lambda, m_{1}, \lambda_{i}, \zeta_{t}^{0}$ \\
Neutron EDM & $\operatorname{Im}\left[\left(\zeta_{t}^{\prime \pm}\right)^{\star}\left(\zeta_{b}^{\prime}\right)^{\star}\right], m_{ \pm}\left(m_{1}, \lambda_{1,2,3}\right)$ \\
$b \rightarrow s \gamma$ & $\operatorname{Re}\left[\left(\zeta_{t}^{\prime \pm}\right)^{\star}\left(\zeta_{b}^{\prime \pm}\right)^{\star}\right],\left|\zeta_{t}^{\prime}\right|^{2}, m_{ \pm}\left(m_{1}, \lambda_{1,2,3}\right)$ \\
$R_{b}$ & $\left|\zeta_{t}^{\prime \pm}\right|^{2}\left|\zeta_{b}^{\prime}\right|^{2}, m_{ \pm}\left(m_{1}, \lambda_{1,2,3}\right)$ \\
EWPD & $m_{1}, \lambda_{1,2,3}$ \\
\hline \hline
\end{tabular}

TABLE I: Summary of constraints.

\section{A. Collider Mass bounds}

The kinematic direct production bound from LEP demands that the sum of new scalar and pseudoscalar Higgs masses obeys $m_{H}+m_{A}>209 \mathrm{GeV}$. This is consistent with the assumption $m_{H}, m_{A}>m_{h}$ made in deriving the Wilson coefficient in Eqn. (11). Moreover

$$
\frac{1}{2}\left(m_{H}^{2}+m_{A}^{2}\right)=m_{1}^{2}+\frac{\lambda_{1}+\lambda_{2}}{2} v^{2}>m_{h}^{2} .
$$


The remaining direct production bound from LEP is on the charged scalar mass, $m_{ \pm}=$ $\sqrt{m_{1}^{2}+\lambda_{1} / 2}>105 \mathrm{GeV}$. This bound is a purely kinematic constraint not dependent on tagging particular final states. ${ }^{7}$

For masses $m_{ \pm}<m_{t}$ the constraints from the Tevatron can also be used. The CDF collaboration [30] constrains $\operatorname{Br}\left(t \rightarrow S^{+} b\right)$ through subsequent decays of $S^{+} \rightarrow c \bar{s}$, while the $\mathrm{D} \phi$ collaboration [31] uses the subsequent decays $S^{+} \rightarrow \tau^{+} \nu_{\tau}$. The latter decay involves the coupling of the charged scalar to leptons, which is not directly related to the parameters of interest. Therefore we use the CDF result which for $m_{s}^{ \pm}<150 \mathrm{GeV}$ gives the constraint

$$
\operatorname{Br}\left(t \rightarrow S^{+} b\right) \operatorname{Br}\left(S^{+} \rightarrow c \bar{s}\right) \lesssim 0.1
$$

For the 2HDM model with $m_{ \pm}<150 \mathrm{GeV}$ we have $\operatorname{Br}\left(S^{+} \rightarrow c \bar{s}\right) \sim 1$ and this becomes

$$
\frac{\left|\zeta_{t}^{\prime \pm}\right|^{2}\left|V_{t b}\right|^{2}}{8 \pi} \frac{\left(m_{t}^{2}-\left(m_{s}^{ \pm}\right)^{2}\right)^{2}}{m_{t}^{3} \Gamma_{t}} \lesssim 0.1
$$

where $\Gamma_{t} \sim 1.3 \mathrm{GeV}$.

\section{B. Consistency Conditions on the Potential}

Although renormalizable, for our purposes this minimal model is best thought of as an effective low energy scalar sector with field content of a UV completion at a relatively low scale $\Lambda \sim \mathrm{TeV}$. We will find that $\mathcal{O}(1)$ parameters are required in the potential which can lead to a relatively low cutoff scale. For consistency we ensure that the couplings of the allowed parameter space do not approach a Landau pole or cause the potential to be unbounded from below up to $\Lambda \sim 1$ $2 \mathrm{TeV} \gg m_{i}$, where $m_{i}$ are the masses in the two scalar doublet model. We impose this constraint considering running and vacuum stability under the complete one-loop RGE flow of the couplings in the potential.

The RGE running of the potential parameters can be derived directly by modifying the effective potential method of [32]. For complex coefficients, within the convention that $S$ is rotated so that $\lambda_{3}$ is real, the scaled beta functions are given in Appendix C. Here the one-loop beta functions are defined in terms of the functions defined above as $\beta_{x}=\hat{\beta}_{x} / 16 \pi^{2}$. In these expressions, $g^{\prime}$ is the

\footnotetext{
${ }^{7}$ The quoted bound of $78.6 \mathrm{GeV}$ at $95 \% \mathrm{CL}$ in [29] is specific to the case $\operatorname{Br}\left(S^{+} \rightarrow \bar{s} c\right)+\operatorname{Br}\left(S^{+} \rightarrow \tau^{+} \nu_{\tau}\right)=1$. We impose the more conservative kinematic bound.
} 
$\mathrm{U}(1)$ gauge coupling $g$ is the $\mathrm{SU}(2)$ coupling of the standard model. The top Yukawa coupling of the Higgs field, and the complex Yukawa couplings of the new doublets are defined as

$$
y_{t}=\sqrt{2} \frac{m_{t}}{v}, \quad \eta_{t}=\zeta_{t}^{0}, \quad \eta_{b}=\zeta_{b}^{0} .
$$

We also need the standard results of the one-loop running of the gauge couplings (above $m_{t}$ ) and the Yukawa couplings; they are

$$
\begin{aligned}
\hat{\beta}_{g^{\prime}}=7 g^{\prime 3}, \quad \hat{\beta}_{g} & =-3 g^{3}, \quad \hat{\beta}_{3}=-7 g_{3}^{3}, \quad \hat{\beta}_{y_{t}}=y_{t}\left[\frac{9}{2} y_{t}^{2}-\frac{17}{12} g^{\prime 2}-\frac{9}{4} g^{2}-8 g_{3}^{2}\right], \\
\hat{\beta}_{\eta_{t, b}} & =\eta_{t, b}\left[\frac{9}{2}\left|\eta_{t}\right|^{2}+\frac{9}{2}\left|\eta_{b}\right|^{2}-\frac{17}{12} g^{\prime 2}-\frac{9}{4} g^{2}-8 g_{3}^{2}\right] .
\end{aligned}
$$

Here we have neglected small mixing effects of the Yukawa coupling operators which we discuss below. For vacuum stability we assume that $\lambda_{6}>0$, which is sufficient for the field space direction along the $S$ axis, considering the masses are constrained to be positive and large due to the bounds discussed above. For directions not along the $h$ or (a particular) $S_{i}$ axis, it is difficult to analytically formulate the conditions for stability when $\lambda_{4,5} \neq 0$, but it is easy to numerically check for runaway behavior, and this is the approach we take.

From [33] we also have the unitary scattering constraint. As we scan parameter space and evolve the couplings of the model under the RGE we insist that the theory remain unitary as the couplings are evolved for mass scales up to the cutoff scale of the theory. However, we find the unitarity constraint has a negligible impact on the parameter space of interest; it is weaker than the demand to avoid Landau poles.

\section{Phenomenological constraints on the loop corrections due to charged scalars}

In this section we consider the constraints arising from virtual scalar exchange contributions to the neutron EDM, $Z \rightarrow \bar{b} b$ and $b \rightarrow s \gamma$. In all of these processes, the dominant contribution comes from charged $S^{ \pm}$exchange because of the fact that the flavor change $t \rightarrow b$ in the loop is not CKM suppressed.

\section{Neutron EDM}

The effect of the exchange of virtual charged and neutral scalars on precision observables leads to significant constraints on models that would otherwise have given rise to successful EWBG. 

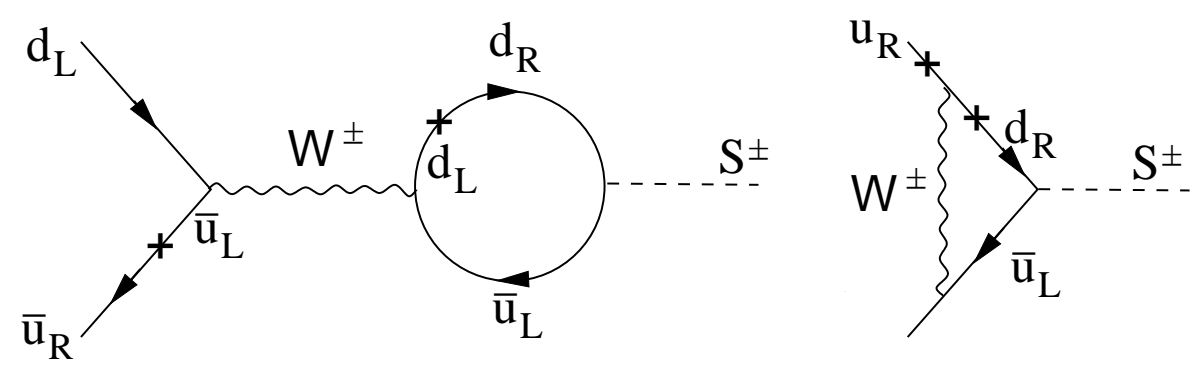

FIG. 1: One loop diagrams that induce an effective local operator of the charged scalar field $s^{ \pm}$to $\bar{u}_{R} d_{L}$ from a coupling to $\bar{u}_{L} d_{R}$. The largest contribution for the diagrams come from a top-bottom quark fermion loop in both cases.

Some electric dipole moment (EDM) constraints on this scenario were discussed in [24]. We focus on the EDM constraint that is not suppressed by small mixing angles or light quark masses that was discussed in [25]. For the neutron EDM we have

$$
\left|\operatorname{Im}\left[\frac{v^{2}\left(\zeta_{t}^{\prime}\right)^{\star}\left(\zeta_{b}^{\prime \pm}\right)^{\star}}{2 m_{t} m_{b}}\right]\right| f_{g}\left(\frac{m_{t}^{2}}{m_{ \pm}^{2}}\right)<0.043, \quad f_{g}(x)=\frac{x \log (x)}{(x-1)^{3}}+\frac{x(x-3)}{2(x-1)^{2}}
$$

Naive Dimensional Analysis (NDA) is used to compute the neutron EDM matrix element in this estimate. ${ }^{8}$ To satisfy this constraint one generically has two choices: the parameter $\zeta_{t}^{ \pm}$can be small, or the relative phases of $\left(\zeta_{t}^{\prime \pm}\right)^{\star}$ and $\left(\zeta_{b}^{\prime} \pm\right)^{\star}$ can be tuned so that the constraint is satisfied. ${ }^{9}$ Either of these choices could conceivably be justified by further model building in the UV.

Taking the parameter $\zeta_{t}^{\prime \pm}$ small to satisfy the EDM bound generally requires $\left|\zeta_{t}^{\prime \pm}\right| \lesssim 10^{-1}$ and the allowed $\zeta_{t}^{\prime \pm}$ decreases as $\zeta_{b}^{\prime \pm}$ increases. One can also accomplish the suppression of $\zeta_{t}^{\prime \pm}$ naturally by using MFV in a model where the new scalar doublet is not a flavour singlet; see [25]. However, there is no exact symmetry in models of this form that distinguishes between the coupling of the charged scalars to $\bar{u}_{R} d_{L}$ and $\bar{u}_{L} d_{R}$; thus the effective parameters $\zeta_{t}^{\prime \pm}$ and $\zeta_{b}^{\prime \pm}$ are not independent and the corresponding operators mix. Therefore the radiative stability of such a choice is an interesting issue. An effective $\zeta_{t}^{\prime \pm}$ is induced through the one-loop diagrams in Fig. 1 from the coupling of the charged scalars to $\bar{u}_{L} d_{R}$ proportional to $\left(\zeta_{b}^{\prime}{ }^{\star}\right)^{\star}$. Calculating these

\footnotetext{
${ }^{8}$ Although the uncertainty in the hadronic matrix element is significant and alternate estimates such as in [34] can weaken this constraint, we conservatively use the NDA estimate in restricting the parameter space.

${ }^{9}$ In general $\left(\zeta_{t}^{\prime \pm}\right)^{\star}$ and $\left(\zeta_{b}^{\prime \pm}\right)^{\star}$ are the combination of many terms that can each individually have an independent phase in the MFV expansion.
} 
diagrams one finds a contribution to the effective $\bar{u}_{R} d_{L}$ Yukawa coupling such that

$$
\begin{aligned}
\delta \zeta_{t}^{\prime \pm} \approx & \frac{\left(\zeta_{b}^{\prime \pm}\right)^{\star} y_{b}}{8 \pi^{2}} \int_{0}^{1} d x(1-x) \log \left[\frac{\mu^{2}}{x m_{t}^{2}+(1-x) m_{b}^{2}}\right] \\
& +\frac{\left(\zeta_{b}^{\prime \pm}\right)^{\star} y_{t}}{128 \pi^{2}} \int_{0}^{1} d x \int_{0}^{1-x} d z\left(-1+(1+x+z) \log \left(\frac{\mu^{2}}{\Delta}\right)\right) \\
& +\frac{\left(\zeta_{b}^{\prime \pm}\right)^{\star} y_{t}}{8 \pi^{2}} \int_{0}^{1} d x \int_{0}^{1-x} d z(1-x-z)\left[\frac{m_{t}^{2}(x+z)^{2}}{\Delta}+\frac{1}{2}\left(1-\log \left(\frac{\mu^{2}}{\Delta}\right)\right)\right]
\end{aligned}
$$

Here $\Delta=-m_{t}^{2}\left(x(1-x)-z^{2}-4 x z-z\right)+m_{b}^{2}\left(2 x z+2 z^{2}-z+x\right)-(1-x-z) M_{W}^{2}$ and we have retained the finite terms of these diagrams to illustrate the effect. For $\zeta_{b}^{\prime \pm} \gtrsim m_{t} / m_{b}$ this contribution to the effective Yukawa coupling is large enough so that the induced effect on $b \rightarrow s \gamma$ and the neutron EDM can be in conflict with experiment even if the coefficient of the operator $\bar{u}_{R} d_{L}$ is chosen to be small at tree level. For this reason we restrict our choice of coupling to $\left|\zeta_{b}^{\prime}{ }^{ \pm}\right| \lesssim 10$ when scanning parameter space.

For EWBG, it is advantageous to satisfy the EDM bound by tuning the relative phases. In this case $\zeta_{t}^{\prime}{ }^{ \pm}>\zeta_{b}^{\prime}$ and one can have a strong coupling of the new Higgs $S$ to the top quark. If on the other hand we were to suppress $\zeta_{t}^{\prime \pm} \sim 10^{-2}$ using the MFV model of [25] or by choosing $\zeta_{t}^{\prime \pm}$ to be small, the small effective top quark coupling to the new field (and new CP violation) suppresses EWBG.

\section{2. $Z \rightarrow b \bar{b}$ constraints}

Another important constraint comes from limits on the charged scalar masses and couplings due to modifications of the $Z b \bar{b}$ vertex. $Z \rightarrow b \bar{b}$ provides direct bounds on the neutral and charged scalars. ${ }^{10}$ The shift in $R_{b}$ due to the virtual corrections of the new scalars comes about through the shift in $\bar{g}_{b}^{R}=\left(\bar{g}_{b}^{R}\right)_{S M}+\delta \bar{g}_{b}^{R}, \bar{g}_{b}^{L}=\left(\bar{g}_{b}^{L}\right)_{S M}+\delta \bar{g}_{b}^{L}$ for the $b$ quark to the $Z$ and we parameterize this effect on $R_{b}$ by

$$
\delta R_{b} \simeq 2 R_{b}\left(1-R_{b}\right)\left(\frac{\delta \bar{g}_{b}^{L}\left(\bar{g}_{b}^{L}\right)_{S M}+\delta \bar{g}_{b}^{R}\left(\bar{g}_{b}^{R}\right)_{S M}}{\left(\bar{g}_{b}^{L}\right)_{S M}^{2}+\left(\bar{g}_{b}^{R}\right)_{S M}^{2}}\right)
$$

We use the predicted SM values $\left(\bar{g}_{b}^{R}\right)_{S M}=0.0774,\left(\bar{g}_{b}^{L}\right)_{S M}=-0.4208$ which gives $R_{b}=$ $0.21578 \pm 0.00010$. Using these results we have

$$
\delta R_{b} \simeq-0.78\left(\delta \bar{g}_{b}^{L}\right)+0.14\left(\delta \bar{g}_{b}^{R}\right) .
$$

$\overline{10}$ See also [35, 36] for recent discussions on $R_{b}$ constraints in models of this form focused on charged scalar exchange. 
Considering that the measured value given by the Particle Data Group is $R_{b}=0.21629 \pm 0.00066$ [37] we have the $1.3 \sigma$ bounds $-1.6 \times 10^{-4}<\delta R_{b}<1.1 \times 10^{-2}$. The shifts in the couplings, where we neglect suppressed corrections of the form $\eta_{i} \lambda_{j} / 16 \pi^{2}$ due to mixing and $m_{b}^{2} / m_{A}^{2}, M_{Z}^{2} / m_{A}^{2}$ suppressed terms are given by

$$
\begin{aligned}
& \delta \bar{g}_{b}^{L} \simeq \frac{\left|\zeta_{t}^{\prime}\right|^{2}}{32 \pi^{2}}\left(\frac{m_{t}^{2} / m_{ \pm}^{2}}{m_{t}^{2} / m_{ \pm}^{2}-1}-\frac{m_{t}^{2} / m_{ \pm}^{2} \log \left(m_{t}^{2} / m_{ \pm}^{2}\right)}{\left(m_{t}^{2} / m_{ \pm}^{2}-1\right)^{2}}\right) \\
& \delta \bar{g}_{b}^{R} \simeq-\frac{\left|\zeta_{b}^{\prime}\right|^{2}}{\left|\zeta_{t}^{\prime}\right|^{2}} \delta \bar{g}_{b}^{L} .
\end{aligned}
$$

Here $s_{W}, c_{W}$ are the sin and cosine of the weak mixing angle. We choose the renormalization scale $\mu=M_{Z}$.

\section{Constraints on $\zeta_{t}^{\prime \pm}$ through $b \rightarrow s \gamma$}

As explained above, we are interested in $\zeta_{t}^{ \pm} \sim 1$ to allow for EWGB, and so we must include terms in the $b \rightarrow s \gamma$ constraint proportional to $\left(\zeta_{t}^{\prime}\right)^{2}$. The constraint from $B R(\bar{B} \rightarrow$ $s \gamma)_{E_{\gamma}>1.6 \mathrm{GeV}}$ at $95 \% \mathrm{CL}$ [38] is given by

$$
-0.42<-\operatorname{Re}\left[\left(\zeta_{t}^{\prime \pm}\right)^{\star}\left(\zeta_{b}^{\prime}\right)^{\star}\right] f_{\gamma}^{(2)}\left(\frac{m_{t}^{2}}{m_{ \pm}^{2}}\right)+\frac{1}{3}\left|\left(\zeta_{t}^{\prime \pm}\right)\right|^{2} f_{\gamma}^{(1)}\left(\frac{m_{t}^{2}}{m_{ \pm}^{2}}\right)<0.12,
$$

where from [39] we have

$$
\begin{aligned}
& f_{\gamma}^{(1)}(x)=\frac{x\left(7-5 x-8 x^{2}\right)}{8(x-1)^{3}}+\frac{3 x^{2}(3 x-2)}{4(x-1)^{4}} \log (x), \\
& f_{\gamma}^{(2)}(x)=\frac{x(3-5 x)}{4(x-1)^{2}}+\frac{x(3 x-2)}{2(x-1)^{3}} \log (x) .
\end{aligned}
$$

\section{Electroweak precision data}

As we scan parameter space we also restrict the mass spectrum of the scalars to be within the 95\% CL region of an electroweak precision data (EWPD) fit. The oblique parameters we use, due to the low mass scale involved, are the STUVWX parameters of [40, 41]. The one loop corrections to the EWPD observables for the 2HDM are standard and are given in [25] (with the appropriate rescaling). The fit we use is described in [42]. We float the Higgs mass at one loop while testing the parameter set against the EWPD constraints and perform a joint fit to the Higgs mass and the new scalar spectrum. To do this we use the self-energy corrections due to the Higgs at one loop 
given by

$$
\begin{aligned}
\Pi_{W W}\left(p^{2}\right) & =\frac{g_{1}^{2}}{16 \pi^{2}}\left[-\frac{A_{0}\left(m_{h}^{2}\right)}{4}-M_{W}^{2} B_{0}\left(p^{2}, m_{h}^{2}, M_{W}^{2}\right)+B_{22}\left(p^{2}, m_{h}^{2}, M_{W}^{2}\right)\right] \\
\Pi_{Z Z}\left(p^{2}\right) & =\frac{g_{1}^{2}}{16 \pi^{2} \cos ^{2} \theta_{W}}\left[-\frac{A_{0}\left(m_{h}^{2}\right)}{4}-M_{Z}^{2} B_{0}\left(p^{2}, m_{h}^{2}, M_{Z}^{2}\right)+B_{22}\left(p^{2}, m_{h}^{2}, M_{Z}^{2}\right)\right] .
\end{aligned}
$$

The one-loop functions used here are defined in [42] as are the STUVWX parameters.

This test is most sensitive to the mass splitting in the scalar spectrum. As the splitting violates custodial symmetry, it leads to important constraints on $\lambda_{3}$. For the MCMC scans we numerically interpolate from a grid scan of EWPD using the $\chi^{2}$ measure defined through the cumulative distribution function for a six-parameter fit. When we float the Higgs mass and scan the parameter space we interpolate between the discrete masses $m_{h}=\{115,130,145,160\} \mathrm{GeV}$. For the new scalar masses we require $m_{H}, m_{A}, m_{ \pm} \leq 700 \mathrm{GeV}$ and vary the masses in this range over the allowed region considering all other consistency constraints on the scalar potential. The MCMC does not show a preference for masses exceeding this $700 \mathrm{GeV}$ upper bound.

Larger Higgs masses $m_{h}$ are allowed in principle in these joint fits due to the positive $\Delta T$ contribution from the mass splitting in the scalar spectrum. We find that successful EWBG prefers a light Higgs.

\section{FINITE TEMPERATURE EFFECTIVE POTENTIAL}

The properties of the electroweak phase transition and the bubble wall profiles needed for computing the baryon asymmetry require knowledge of the effective potential $V_{\text {eff }}(H, S)$ at finite temperature. We compute it at one-loop order, along with the corresponding zero-temperature loop correction. The full potential can be expressed as

$$
V_{\text {eff }}=V_{\text {Tree }}+V_{\mathrm{CW}}+V_{\mathrm{CT}}+V_{\mathrm{T}}
$$

where $V_{\text {Tree }}$ is given by (6), $V_{\mathrm{T}}$ is the thermal contribution, $V_{\mathrm{CW}}$ is the one-loop Coleman-Weinberg (CW) potential, and $V_{\mathrm{CT}}$ are counterterms, which for convenience can be chosen so as to maintain various tree-level relations for the scalar mass eigenvalues and VEVs.

The CW potential is given by

$$
V_{\mathrm{CW}}=\sum_{i} \pm \frac{g_{i}}{64 \pi^{2}} m_{i}^{4}\left(\ln \frac{m_{i}^{2}}{Q^{2}}-\frac{3}{2}\right)
$$


where $g_{i}$ is the multiplicity of species $i, m_{i}$ is its field dependent mass and + sign is for bosons and - sign for fermions. The field-dependent masses are given in Appendix B, $Q$ is a renormalization scale, which we take to be $v / \sqrt{2}$, where $v=246 \mathrm{GeV}$ is the Higgs VEV. Some residual $Q$ dependence remains in our results since we do not perform a complete renormalization at 1-loop level including wave function renormalization. We include the Higgs bosons and electroweak gauge bosons in the sum, as well as the top quark. We work in Landau gauge where the FadeevPopov ghosts decouple at the one-loop level.

The effective potential is a function of two complex fields, $H^{0}$ and $S^{0}$, but we have the freedom to remove the phase of $H^{0}$ (the Goldstone boson mode) by making the appropriate SU(2) gauge transformation on both fields. Doing so simplifies our task by eliminating the unphysical degree of freedom. We thus regard $V_{\text {eff }}$ as a function of the three fields $h, s_{R}, s_{I}$ as defined below Eqn. (77): $H^{0} \equiv \frac{1}{\sqrt{2}} h$ and $S^{0} \equiv \frac{1}{\sqrt{2}}\left(s_{R}+i s_{I}\right)$.

\section{A. Counterterms and Goldstone bosons}

For convenience we introduce counterterms to preserve the tree-level relationships for masses and VEVs in the zero-temperature potential. In the $\left\{h, s_{R}, s_{I}\right\}$ field basis we thus demand that $\partial V / \partial h=\partial V / \partial s_{R}=\partial V / \partial s_{I}=0$ at $h=v, s_{R}=s_{I}=0$. Denoting the tree-level VEVs by $\left\langle\phi_{i}\right\rangle$, this requires

$$
\left.\frac{\partial V_{\mathrm{CT}}}{\partial \phi_{i}}\right|_{\left\langle\phi_{i}\right\rangle}=-\left.\frac{\partial V_{\mathrm{CW}}}{\partial \phi_{i}}\right|_{\left\langle\phi_{i}\right\rangle}
$$

Similarly, to preserve the mass relations we require that

$$
\left.\frac{\partial^{2} V_{\mathrm{CT}}}{\partial \phi_{i} \partial \phi_{j}}\right|_{\left\langle\phi_{i}\right\rangle}=-\left.\frac{\partial^{2} V_{\mathrm{CW}}}{\partial \phi_{i} \partial \phi_{j}}\right|_{\left\langle\phi_{i}\right\rangle},
$$

where $\phi_{i}=h, s_{R}, s_{I}$. There is a problem in principle with carrying out (41). We work in the Landau gauge where ghosts decouple but Goldstone boson (GB) contributions are retained in the sum over species. In particular, intermediate GBs contribute to the mass of the Higgs, and formally their contribution to $\partial^{2} V_{\mathrm{CW}} / \partial h^{2}$ is IR log divergent due to terms of the form $\left(\partial m_{g b}^{2} / \partial h\right)^{2} \ln m_{g b}^{2}$, where the prefactor does not vanish at the VEVs, but the argument of the log does. This shows that renormalizing the Higgs mass at zero external momentum with massless Goldstone modes is not a well defined procedure. Some authors choose to simply omit the GB contributions to the effective potential, but in the present study we find that this is not justified. Of course one can renormalize 
the Higgs mass at any momentum scale $p^{2} \neq 0$ and a consistent procedure of renormalizing onshell at $p^{2}=m_{h}^{2}$ was implemented in ref. [43]. This cures the GB-problem because the external Higgs on-shell momentum flows through the loop of internal GBs contributing to the Higgs selfenergy and cuts off the IR divergence that occurs when renormalizing at $p^{2}=0$, Eqn. (41). The effect can be approximately captured by replacing $m_{g b}^{2} \rightarrow m_{\mathrm{IR}}^{2}$ in (41) for the determination of the counterterms, where $m_{\mathrm{IR}}^{2}$ is some IR cutoff. Since the IR divergence is only logarithmic, the effective potential is not greatly sensitive to the exact value. We find that $m_{\mathrm{IR}}^{2} \cong m_{h}^{2}$ gives a good approximation to the more exact prescription of ref. [43]. This is the procedure we adopt.

In practice, not all possible counterterms are required. This is because we do not expect to be able to measure all of the couplings in the scalar potential in the near future, and therefore we do not need to relate all of them (such as $\lambda_{6}$ ) to observables. For our purposes, it is sufficient to include nine counterterms,

$$
\begin{aligned}
V_{\mathrm{CT}} & =\frac{1}{16} \delta \lambda h^{4}-\frac{1}{2} \delta \mu^{2} h^{2}+\frac{1}{2} \delta m_{1}^{2}\left(s_{R}^{2}+s_{I}^{2}\right)+h\left(\delta m_{2 R}^{2} s_{R}-\delta m_{2 I}^{2} s_{I}\right) \\
& +\frac{1}{2} \delta \lambda_{3}^{R} h^{2}\left(s_{R}^{2}-s_{I}^{2}\right)-\delta \lambda_{3}^{I} h^{2} s_{R} s_{I}+\frac{1}{2} h^{3}\left(\delta \lambda_{5}^{R} s_{R}+\delta \lambda_{5}^{I} s_{I}\right)
\end{aligned}
$$

which are fixed by the nine independent renormalization conditions set by eqs. 40,41). Explicit formulas for the counterterm couplings defined in this way are given in Appendix A.

\section{B. Thermal corrections}

The unimproved pure thermal contribution to $V_{\text {eff }}$ is given by:

$$
V_{\mathrm{T}}=\frac{T^{4}}{2 \pi} \sum_{i} \pm g_{i} \int_{0}^{\infty} d x x^{2} \ln \left(1 \mp \exp \left(-\sqrt{x^{2}+m_{i}^{2}\left(H^{0}, S^{0}\right) / T^{2}}\right),\right.
$$

where upper signs are for bosons and lower signs for fermions. Splitting Eqn. (43) into distinct sums over bosonic and fermionic species and expanding it to order $O\left(m_{i}^{6} / T^{2}\right)$ one finds (see for example ref. [44]):

$$
\begin{aligned}
V_{T} \cong c T^{4} & +\sum_{F} g_{F}\left[\frac{m_{F}^{2} T^{2}}{48}-\frac{m_{F}^{4}}{64 \pi^{2}}\left(\ln \frac{m_{F}^{2}}{T^{2}}-c_{F}\right)\right] \\
& +\sum_{B} g_{B}\left[\frac{m_{B}^{2} T^{2}}{24}-\frac{m_{B}^{3} T}{12 \pi}+\frac{m_{B}^{4}}{64 \pi^{2}}\left(\ln \frac{m_{B}^{2}}{T^{2}}-c_{B}\right)\right] .
\end{aligned}
$$

The lowest order potential (43) can be improved by resumming diagrams corresponding to insertions of the thermal mass corrections,

$$
m_{i}^{2}\left(H^{0}, S^{0}\right) \rightarrow m_{i}^{2}\left(H^{0}, S^{0}\right)+\delta m_{i}^{2}
$$


where $\delta m_{i}^{2}$ is of the form $T^{2}$ times coupling constants. The Debye mass matrices can be obtained by computing the thermal self-energies in the high-temperature limit, but they can also be directly inferred from (43). For a bosonic degree of freedom $\phi_{i}$ the Debye mass matrix is

$$
\delta m_{i j}^{2}=\sum_{k} \frac{g_{k}}{24} \frac{\partial m_{k}^{2}}{\partial \phi_{i} \partial \phi_{j}} T^{2}
$$

where $k$ runs over the relevant bosons and fermions in the theory, and $m_{k}^{2}$ are the field dependent masses given in Appendix $\mathbb{B}^{11}$. Fermions do not get a thermal correction. The thermally corrected mass $m_{i}^{2}+\delta m_{i}^{2}$ in (45) denotes an eigenvalue of the full mass matrix $m_{i j}^{2}+\delta m_{i j}^{2}$.

Strictly speaking the ring-improvement is a consistent correction only for the bosonic zero modes in the high temperature limit $m / T \ll 1$ [44], correcting the masses only in the cubic terms in the bosonic expansion in (44). However, we need the effective potential also for large $m / T$, and we need to be able to smoothly connect the two regimes. In the $m / T \gtrsim 1$ regime a cubic term with eigenvalues computed from high- $T$ Debye mass corrections (46) becomes a poor approximation for the effective potential. The situation could be remedied by computing the Debye masses for arbitrary $m / T$, whence the Debye corretions would be exponentially suppressed at large $m / T$. This would be a lengthy computation but luckily a more convenient and mathematically equally consistent prescription is available: one implements (45) with the high-T expressions (46) directly in (43) without making any high-temperature expansion [45]. Both approaches give essentially indistinguishable results in the high- $T$ limit while the latter prescription also smoothly connects to the correct (vanishing) thermal correction at $m / T \gg 1$.

To complete our approximation we also make the Debye correction within the 1-loop vacuum correction $V_{\mathrm{CW}}$. Indeed, it is known that at 1-loop level the $\ln \left(m_{i}^{2}\right)$ terms cancel between the $V_{\mathrm{CW}}$ and the high- $T$ expansion of $V_{\mathrm{T}}$ and we ought to preserve this property in our ring-improved potential. Log-terms corrected in this way do not induce spurious nonanalytic behaviour associated with the negative values of $m_{i}^{2}$, which frequently occur for the Goldstone modes as well as for the physical Higgs in regions where the curvature of the potential is negative. Negative $m_{i}^{2}$ induce an imaginary part of the effective potential due to the nonanalytic cubic $T\left(m^{2}\right)^{3 / 2}$ term, signalling an instability of homogenous zero-modes. Here we are only concerned with the real part of the potential obtained by replacing the cubic term with $T\left|m^{2}\right|^{3 / 2}$. Numerically we use the high- $T$ expansion where $m / T$ is sufficiently small and smoothly match it onto a numerical fit to an exact

\footnotetext{
${ }^{11}$ The sum over a given scalar of fermion representation can be written as $\sum_{k} g_{k} m_{k}^{2}=\operatorname{Tr}\left[m m^{\dagger}\right]$.
} 
thermal integral for larger $m / T$.

\section{ELECTROWEAK PHASE TRANSITION}

We wish to identify models which have a first order electroweak phase transition, and which satisfy the constraint $v_{c} / T_{c}>1$ for the Higgs VEV and critical temperature when bubbles of the broken phase nucleate. This constraint ensures that any baryons created during the phase transition are not significantly depleted by residual sphaleron interactions inside the bubbles.

\section{A. Finding $T_{c}$}

To determine the strength of the phase transition, we use the following algorithm. At sufficiently high temperatures one can usually find, through numerical minimization, a minimum of the potential that is in or close to the symmetric vacuum with vanishing Higgs VEVs. (We discuss exceptions to this statement below). At low temperatures one can of course find the broken minimum with $\left\langle H^{0}\right\rangle \cong v / \sqrt{2}$. The first step is to try to bracket the critical temperature $T_{c}$ of the phase transition by subdividing an initial interval $\left(T_{1}, T_{2}\right)$ in the middle, $T_{m}=\frac{1}{2}\left(T_{1}, T_{2}\right)$. If only the symmetric minimum exists at $T_{m}$, then we replace $T_{1}$ by $T_{m}$, whereas if only the broken minimum exists, we replace $T_{2}$ by $T_{m}$. Continuing in this way, we will either reach an interval over which both local minima simultaneously exist, in which case we have bracketed $T_{c}$ for a first order phase transition, or else the width of the interval becomes smaller than some cutoff (we take $0.1 \mathrm{GeV}$ ), and the transition is deemed to be second order. Once $T_{c}$ is bracketed, one can accurately find $T_{c}$ and $v_{c}$ by minimizing the function $f(T)=\left|V_{\mathrm{sym}}-V_{\mathrm{br}}\right|$, where $V_{\mathrm{sym}}$ and $V_{\mathrm{br}}$ are respectively the values of the potential at the symmetric and broken minima.

We find that for nonzero values of $\lambda_{4}$, the "symmetric" minimum in fact sometimes has a sizeable VEV $v_{s}=\sqrt{2\left(\left|\left\langle H^{0}\right\rangle\right|^{2}+\left|\left\langle S^{0}\right\rangle\right|^{2}\right)}$. As long as $v_{s}$ is small, sphalerons in the quasisymmetric phase are still fast enough to induce baryogenesis. However if $v_{s}$ is too large, the sphaleron interactions will be suppressed in front of the bubble wall and lead to a suppressed baryon asymmetry. We take the upper bound to be $v_{s} / T_{c}<0.4$. This is the point at which the sphaleron rate in the "symmetric" phase starts to become significantly less than its value at $v_{s}=0$, as can be seen from Eqn. (67) below. An example is provided by the parameter set $m_{h}=122$ $\mathrm{GeV}, m_{1}=185.4 \mathrm{GeV}, m_{2}=0, \lambda_{1}=1.335, \lambda_{2}=2.553, \lambda_{3}=-1.291, \lambda_{4}=-0.114+0.434 i$, 


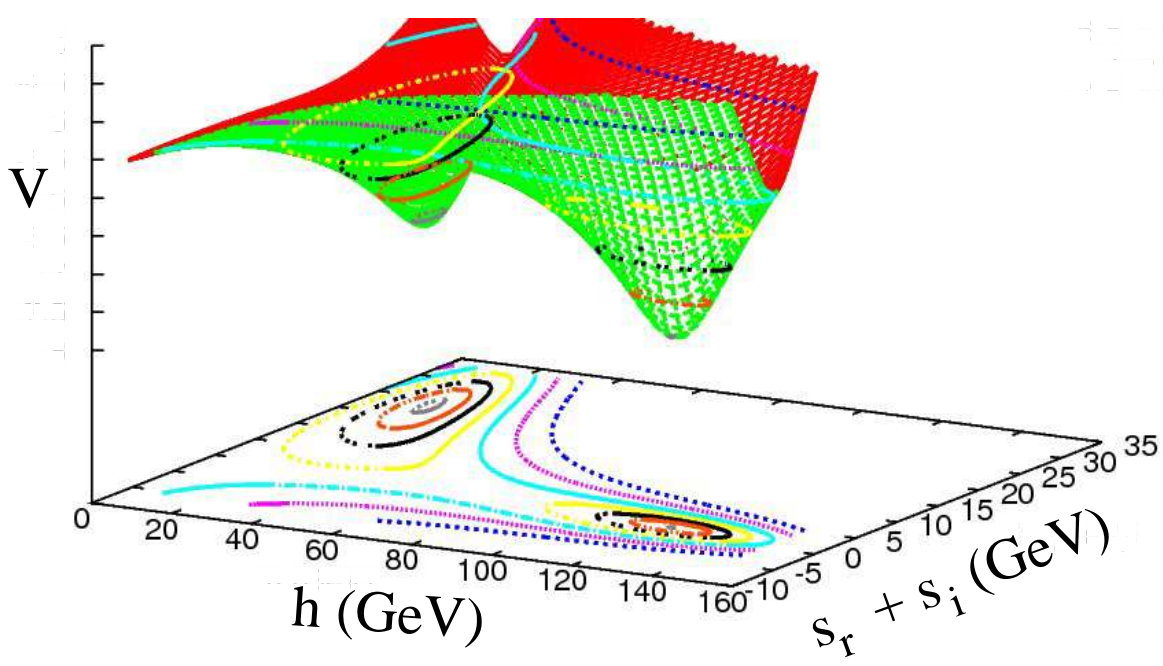

FIG. 2: Potential at $T=T_{c}$ for a case that exhibits mild electroweak symmetry breaking in the quasisymmetric minimum. The log of [V minus a constant near the minimum value] is plotted to exaggerate the barrier between the two minima.

$\lambda_{5}=0, \lambda_{6}=1, \eta_{t}=-0.096$. The potential at the critical temperature is shown in Fig. 2. It has $v_{s} / T_{c}=0.34$, and so still satisfies our criterion for sphaleron interactions being fast enough in the symmetric phase.

\section{B. Search strategy}

The scalar potential of the general two Higgs doublet model we investigate has many parameters. While it is possible to scan them on a coarse grid to find many examples satisfying the particle physics contstraints discussed in section III, very few of these models also have a strong enough phase transition, with $v_{c} / T_{c}>1$. This is not surprising, since imposing constraints lowers the dimensionality of the allowed parameter space. For example, searching for points in an 12D space that happen to lie on a complicated 8D subsurface is bound to be inefficient if we restrict the search to points on a necessarily coarse grid in the full 12D space. Such a search could be made more efficient if we had an analytic formula for the constraint, but this is not possible for the EWPD. Table $\llbracket$ shows the results of such grid searches for several values of $m_{h}$. Later we must impose the additional requirement of getting a large enough baryon asymmetry, which is difficult to achieve. It is therefore desirable to maximize the number of models that satisfy the present constraints. 


\begin{tabular}{|c|c|c|}
\hline$m_{h}(\mathrm{GeV})$ & $\#$ models & \# strong EWPT \\
\hline 115 & 210,000 & 92 \\
120 & 195,000 & 49 \\
130 & 171,000 & 25 \\
\hline
\end{tabular}

TABLE II: Results of grid searches of the 2HDM parameter space, showing the numbers of models that satisfy particle physics constraints, and of these the number having $v_{c} / T_{c}>1$.

A more efficient search strategy is the Markov Chain Monte Carlo (MCMC) algorithm. Instead of blindly searching on a grid, this method favors points with a stronger phase transition, while still attempting to broadly explore the space. Starting at some point $\left\{\lambda_{i}\right\}$ in the parameter space, a chain of parameter sets is accumulated. A trial step is taken from the current point by varying the $\lambda_{i}$ randomly, with step sizes taken from a Gaussian distribution whose variance can be tuned for optimal performance. Let $r$ be the ratio $\left(v_{c} / T_{c}\right)_{\text {new }} /\left(v_{c} / T_{c}\right)_{\text {old }}$, comparing the previous point to the new one. The new point is accepted into the chain if it has $r>1$, and it is accepted with probability $r$ if $r<1$. In this way, a chain of models is obtained, whose probability distribution for $v_{c} / T_{c}$ is proportional to $v_{c} / T_{c}$, thus favoring larger values of $v_{c} / T_{c}$. The occasional acceptance of points with smaller values of $v_{c} / T_{c}$ is helpful to avoid getting stuck in a local extremum of the probability distribution, so that the parameter space is broadly explored.

We modify the basic MCMC procedure by also rejecting the trial point if it has an unstable vacuum, or if it fails to satisfy any of the particle physics constraints described in section III. We also adopt a trick to boost the acceptance rate: the trial point is alternately generated using the last point or the next-to-last point in the chain. Presumably the increase in acceptance results from the fact that the next-to-last point has already succeeded in leading to one new point in the chain. We set the Gaussian widths for the randomly generated steps to $10 \%$ of the current value for the parameters $\lambda_{1}, \lambda_{2}, \lambda_{3}$ and $\sqrt{\left|m_{1}^{2}\right|}$, and to 0.1 for the other dimensionless parameters $\lambda_{4}^{R}, \lambda_{4}^{I}, \lambda_{6}$, $\zeta_{b}^{0}, \zeta_{t}^{0}$ and the phase $\phi$ of $\zeta_{t}^{0}$. The width for steps in the mass of the Higgs is $5 \mathrm{GeV}$.

The above description holds if one is only interested in generating models with large $v_{c} / T_{c}$. We would also like to favor models in which the baryon asymmetry can be large. Therefore we additionally compute the change of the CP-violating phase of the top quark mass across the bubble wall $\left(m_{t}(z)=\left|m_{t}(z)\right| e^{i \theta(z)}\right)$,

$$
\Delta \theta=\left.\theta(z)\right|_{-\infty} ^{\infty}
$$


and test the quantity $(\Delta \theta)\left(v_{c} / T_{c}\right)$ instead of just $v_{c} / T_{c}$. It then becomes consistent, within the philosophy of the MCMC approach, to impose $v_{c} / T_{c}>1$ as another constraint to be satisfied by all models in the chain. Similarly, to search for models that lead to successful baryogenesis as well as the $D \emptyset$ dimuon anomaly, we take $(\Delta \theta)\left(v_{c} / T_{c}\right)\left|\zeta_{b}^{\prime} 0 /\left(V_{t b}^{\star} V_{t s}\right)\right|$ as the quantity to be maximized by the MCMC.

We have noticed that having nonzero $\left|\lambda_{5}\right|$ tends to weaken the phase transition. (Recall that $\lambda_{5}$ and $m_{2}^{2}$ are linked to each other by our choice of field basis in which $\langle s\rangle=0$ at zero temperature, insured by Eqn. (77.) This can be understood analytically by considering a simplified version of the potential,

$$
V \sim V_{h}(h)+\frac{1}{2} m_{1}^{2} s^{2}+\lambda_{5} s h\left(h^{2}-v^{2}\right)
$$

Upon integrating out $s$, it becomes

$$
V \sim V_{h}(h)-\frac{\lambda_{5}^{2}}{m_{1}^{2}} h^{2}\left(h^{2}-v^{2}\right)^{2}
$$

The second term always has positive curvature in the region $h \sim v$, tending to cancel out any barrier between the symmetric and broken phases that might arise due to finite temperature effects. We have compared MCMC runs in which $\lambda_{5}$ is fixed at zero to those where it is allowed to vary and find no significant difference with respect to the frequency of finding a given value of the baryon asymmetry.

\section{Results}

To illustrate some of the statistics of models with a strong phase transition, we consider a chain of $10^{4}$ models that pass all of the constraints. In this section and in section VI we use $\left|\eta_{D}^{\prime}\right|$ as a more convenient parametrization for $\zeta_{b}^{\prime 0}$ and similarly $\left|\eta_{U}\right|$ for $\zeta_{t}^{0}$ to avoid having to rescale by CKM factors and Yukawa couplings in the MCMC. (The relation between the $\eta_{Q}$ and $\zeta_{q}$ parameters was given in section IID.) We do not insist upon the dimuon anomaly here, but we allow $\left|\eta_{D}^{\prime}\right|$ to vary so that we can check a posteriori what fraction of otherwise allowed models can be compatible with this potential constraint. We take the parameter $f=1$ in eq. (24) so that the strength of the neutron EDM and $b \rightarrow s \gamma$ are at a typical level given the assumed value of $\eta_{D}^{\prime}$. Fig. 3 (left panel) shows the distributions of $m_{h}$, the new scalar masses $m_{H}, m_{A}, m_{ \pm}$(where $m_{H / A}$ were defined in Eqn. (9) and $m_{ \pm}$are the charged Higgs masses), the dimensionless couplings $\left|\lambda_{4}\right|,\left|\lambda_{5}\right|, \lambda_{6},\left|\eta_{D}^{\prime}\right|$ and $\left|\eta_{U}\right|$, and the quantities to be maximized (in addition to $\left|\eta_{D}^{\prime}\right|$ ): $\Delta \theta$ (Eqn. (47)) and $v_{c} / T_{c}$. One 

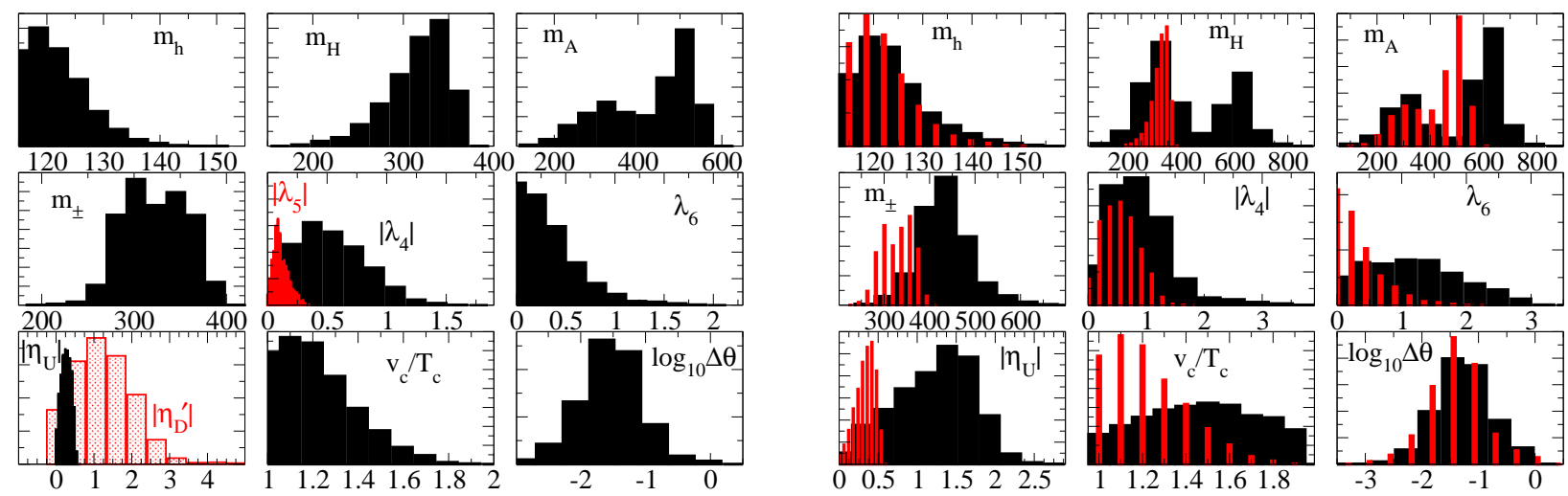

FIG. 3: Left: distributions of parameters satisfying sphaleron and particle physics bounds, including $\zeta_{b}^{\prime} 0$ and $\zeta_{b}^{\prime} \pm$, but not insisting on reproducing the magnitude of the observed $\mathrm{D} \phi$ dimuon excess. $m_{H, A}$ denote masses of the new scalar and pseudoscalar Higgs bosons, respectively. Normalizations are arbitrary. Masses are in GeV. Right: distributions from MCMCs in which $\zeta_{b}^{\prime 0}=\zeta_{b}^{\prime \pm} \approx 0$, and either omitting (heavy black bars) or applying (narrow red bars) the constraints from EWPD, $b \rightarrow s \gamma$, neutron EDM, and perturbativity of couplings. Here $\left|\eta_{U}\right|,\left|\eta_{D}^{\prime}\right|$ are proxies for $\left|\zeta_{t}^{0}\right|,\left|\zeta_{b}^{\prime}\right|$.

sees that relatively low Higgs masses $m_{h}<140 \mathrm{GeV}$ are necessary, the masses of the new scalars are less than $350-500 \mathrm{GeV}$ for $s_{R}$ with mass $M_{H}$ and the charged scalars (the pseudoscalar $s_{I}$ with mass $M_{A}$ tends to be heavier, $m_{A} \lesssim 500 \mathrm{GeV}$ ), and dimensionless couplings are typically less than 1 in magnitude with the exception of $\left|\eta_{D}^{\prime}\right|$, which we have deliberately pushed toward larger values in the MCMC. This parameter has no direct impact on the phase transition dynamics (since we do not include the $b$ quark contributions to $V_{\text {eff }}$ ), but it does play a role in the $R_{b}, b \rightarrow s \gamma$, EDM and RGE constraints.

To appreciate the impact of the new particle physics constraints we have imposed relative to previous studies, we also generated a chain of models that satisfy only the $v_{c} / T_{c}>1$ requirement and the accelerator bounds on masses. The resulting parameter distributions are shown in the right panel of Fig. 3. They are much broader than those which incorporate the constraints. This explains in part why we find it more difficult to achieve enough baryogenesis in the 2HDM compared to earlier investigations. But there are other important reasons having to do with the production mechanism, as we describe in the next section. 


\section{BARYOGENESIS}

To find the baryon asymmetry for a given model, once it has been established to give a first order phase transition and $T_{c}$ has been determined, several steps must be taken. (1) The solution $\left\{h(z), s_{R}(z), s_{I}(z)\right\}$ for the fields in the bubble wall must be constructed. We will refer to it as the path taken by the wall in field space. (2) A set of coupled Boltzmann transport equations must be solved for the chemical potentials $\mu_{i}(z)$ and velocity perturbations $u_{i}(z)$ of the particle species considered to be most relevant for creating a local CP asymmetry in the vicinity of the wall. (3) Some linear combination of the $\mu_{i}$ gives the chemical potential for left-handed baryon number, $\mu_{B_{L}}$, which biases sphaleron reactions in the symmetric phase in front of the wall. The integral of $\mu_{B_{L}}$ in the symmetric phase region gives the baryon asymmetry, up to some proportionality factor.

Our procedure is to carry out these steps for each model in the chains produced by the MCMC. We recall that these are models passing all the phenomenological constraints as well as the sphaleron bound $v_{c} / T_{c}>1$, and also tending to have a large phase change $\Delta \theta$, and optionally large values of $\zeta_{b}^{\prime 0}$. We weed out the models that necessarily give a small baryon asymmetry using a predictor. Statistically the dimensionless quantity

$$
q \equiv \frac{v_{c}}{T_{c}} \frac{\Delta \theta}{L_{w} T_{c}}
$$

where $L_{w}$ is the bubble wall thickness, tends to be proportional to the maximum value of the baryon asymmetry that can be produced. The correlation is shown in Fig. 4. There are several reasons that the actual baryon asymmetry can fall below the maximum value predicted by $q$ (as we will discuss below), but large values of $n_{B}$ almost always require that $q \gtrsim 0.15\left(n_{B} / n_{B \text {,obs }}\right)$. Therefore if one wants to find the instances in a chain of 10,000 models that give the largest baryon asymmetry, it is not necessary to compute $n_{B}$ for all of them, but rather focus on those with the largest $q$ values. Of course, $q$ is much faster to compute than is $n_{B}$.

\section{A. Bubble wall path and the CP-violating phase of the top quark}

In this section we discuss our procedure for finding the profiles of the fields $h, s_{R}, s_{I}$ in the bubble walls, needed for computing baryon production. In principle, this task is complicated because the true bubble nucleation temperature $T_{\text {nuc }}$ is somewhat lower than the critical temperature $T_{c}$. A complete account of the phase transition characteristics would involve finding $T_{\text {nuc }}$, computing the latent heat that drives the transition and evaluating the frictional forces exerted by the particles 


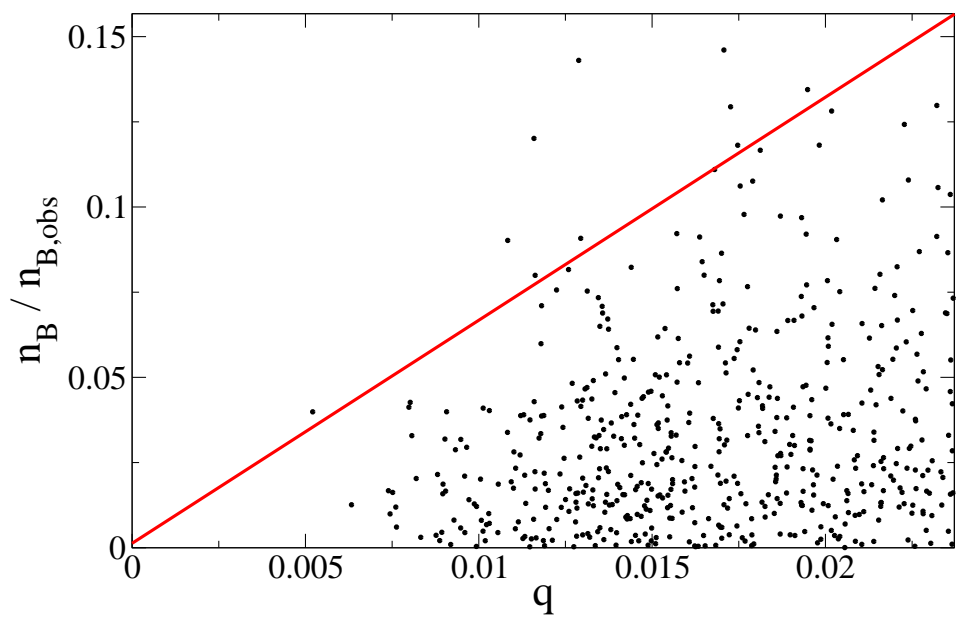

FIG. 4: Scatter plot of baryon number in units of the observed density $\left(n_{B} / n_{B, \text { obs }}\right)$ versus the predictor $q$, Eqn. (50).

and fields on the expanding wall. Such a calculation would yield both the wall velocity and the Higgs field profiles over the transition region, which is what we need for a baryogenesis calculation. This would be a difficult task however, and its results would still be uncertain because, e.g., reliable techniques to compute the interactions of the infrared gauge-field modes with the bubble wall do not exist. However, generically $T_{c}-T_{\text {nuc }} \ll T_{c}$ so that $V_{\text {eff }}$ changes little between $T_{c}$ and $T_{\text {nuc }}$ and one might expect that the true profiles are reasonably well approximated by the solution interpolating between the degenerate minima at $T=T_{c}$. This is the approximation we shall adopt here, and the wall veolocity $v_{w}$ is left as an external free parameter. The wall profiles are then found by numerically solving the equations of motion

$$
\frac{\partial^{2} \phi_{i}}{\partial z^{2}}=-\frac{\partial V_{\mathrm{eff}}}{\partial \phi_{i}}
$$

at the critical temperature, where $z$ is the direction transverse to the wall, subject to the boundary conditions that the fields approach the broken or symmetric minima as $z \rightarrow \pm \infty$.

\section{Gauge dependence of bubble wall profiles}

The coupling between the neutral scalars and the $Z$-field poses a complication in solving for the bubble wall profiles. This is because the covariant derivative terms $\left|D_{z} \phi_{i}\right|^{2}$ provide a source for the classical $Z$-field, and so one is faced by the problem of minimizing the Hamiltonian:

$$
\int \mathrm{d} z\left(\left|D_{z} H^{0}\right|^{2}+\left|D_{z} S^{0}\right|^{2}+V_{\text {eff }}\left(H^{0}, S^{0}, T_{c}\right)+\frac{1}{2} M_{Z}^{2} Z_{\mu} Z^{\mu}+\ldots\right)
$$


where $D_{z}=\partial_{z}-i\left(g / 2 \cos \theta_{W}\right) Z_{z}$ and dots indicate other $Z$-dependent terms. Writing $\sqrt{2} H^{0} \equiv$ $h e^{i \varphi_{h}}$ and $\sqrt{2} S^{0} \equiv s e^{i \varphi_{s}}$ and using the fact that the effective potential depends only on the phase difference $\varphi_{h}-\varphi_{s}$ one can show that

$$
\partial_{\mu}\left(j_{Z}^{\mu}-M_{Z}^{2} Z^{\mu}\right)=0
$$

where $M_{Z}^{2}=g^{2}\left(h^{2}+s^{2}\right) /\left(4 \cos \theta_{W}^{2}\right)$ and $j_{Z}^{\mu}$ is the current sourcing the $Z$-field:

$$
j_{Z}^{\mu} \equiv \frac{g}{2 \cos \theta_{W}}\left(h^{2} \partial^{\mu} \varphi_{h}+s^{2} \partial^{\mu} \varphi_{s}\right) .
$$

Since all classical fields should vanish far away from the wall, we find that

$$
Z^{z}(z)=\frac{j_{Z}^{z}}{M_{Z}^{2}(z)}
$$

while all other components vanish. Recall that in our formulation of the effective potential, we have chosen a gauge where the Goldstone boson vanishes, hence $\varphi_{h} \equiv 0$. This leads to a nonvanishing source (54) for the $Z$-field since $\varphi_{s}$ is generally nonzero. Of course $Z$-field is gaugedependent, and by an alternative choice of the basis for phases [46] we could make $j_{Z}^{\mu} \equiv 0$, and in this gauge $Z^{\mu} \equiv 0$, by Eqn. (55). The price to pay for this simplicity is that one would have to keep track of four fields in the effective potential rather than three.

\section{Spatially varying phase of $m_{t}$}

A key quantity for computing the baryon asymmetry in the current scenario is the spatially varying CP-violating phase of the top quark mass, given a priori by

$$
m_{t}(z)=\frac{y_{t}}{\sqrt{2}} e^{i \varphi_{h}}\left(h+\frac{\zeta_{t}^{0}}{y_{t}} s e^{i \varphi_{s h}}\right),
$$

where $\varphi_{s h} \equiv \varphi_{s}-\varphi_{h}$. As we have observed, because of the $\mathrm{U}(1)_{Y}$ gauge symmetry, $\varphi_{h}$ and $\varphi_{s}$ are not both physically meaningful quantities, so it is interesting to see how both gauges discussed above lead to the same phase for the top quark mass (especially since different authors have chosen different gauges in previous treatments). First, in the $Z \equiv 0$ gauge we can solve for the phase $\varphi_{h}$ using the constraint $j_{Z}^{\mu}=0$ :

$$
\partial_{z} \varphi_{h}=-\frac{s^{2}}{h^{2}+s^{2}} \partial_{z} \varphi_{s h}
$$

$\varphi_{h}$ can be reconstructed after the fields $h, s$ and $\varphi_{s h}$ are determined using equations following from the reduced Hamiltonian:

$$
\int \mathrm{d} z\left(\frac{1}{2}\left(\partial_{z} h\right)^{2}+\frac{1}{2}\left(\partial_{z} s\right)^{2}+\frac{1}{2} \frac{s^{2} h^{2}}{s^{2}+h^{2}}\left(\partial_{z} \varphi_{s h}\right)^{2}+\tilde{V}_{\text {eff }}\left(h, s, \varphi_{s h}, T_{c}\right)\right)
$$


where $\tilde{V}\left(h, s, \varphi_{s h}\right)=V_{\text {eff }}\left(h, s_{R}=s \cos \theta_{s h}, s_{I}=s \sin \theta_{s h}, T_{c}\right)$. (We can always shift $\varphi_{h}$ into the phase of $S$ in $V_{\text {eff }}$ because it is invariant under global $\mathrm{SU}(2) \times \mathrm{U}(1)_{y}$ transformations, and contains no derivatives of the fields.)

Alternatively, if one sets $\varphi_{h} \equiv 0$, it is necessary to deal with the nonvanishing $Z_{\mu}$-field:

$$
Z_{z}(z)=\frac{g}{2 \cos \theta_{W} M_{Z}^{2}} s^{2} \partial_{z} \varphi_{s}
$$

This field induces an additonal CP-violating force acting on the top quark due to the gauge interaction $\left(g / 2 \cos \theta_{W}\right) \bar{t} \not \gamma_{5} t$. This term can be removed by a local axial transformation $t \rightarrow e^{i \gamma^{5} \varphi_{Z} / 2} t$, which however reintroduces an additional overall phase $\varphi_{Z}$ into $m_{t}$ :

$$
m_{t}(z) \rightarrow \frac{y_{t}}{\sqrt{2}} e^{i \varphi_{Z}}\left(h+\frac{\zeta_{t}^{0}}{y_{t}} s e^{i \varphi_{s}}\right) .
$$

It is easy to see that this transformation removes the interaction term with $Z_{z}$ given by Eqn. (59) only if $\partial_{z} \varphi_{Z}=-\frac{s^{2}}{h^{2}+s^{2}} \partial_{z} \varphi_{s}$ which is identical to Eqn. (57) in the gauge $\varphi_{h}=0$. Denoting $\zeta_{t}^{0} / y_{t}=\left|\zeta_{t}^{0} / y_{t}\right| e^{i \varphi_{\eta}}$, we find that the total phase of $m_{t}$ is given by

$$
\theta(z)=\varphi_{h}(z)+\tan ^{-1}\left(\frac{\left|\frac{\zeta_{t}^{0}}{y_{t}}\right| s \sin \left(\varphi_{\eta}+\varphi_{s h}\right)}{h+\left|\frac{\zeta_{t}^{0}}{y_{t}}\right| s \cos \left(\varphi_{\eta}+\varphi_{s h}\right)}\right) .
$$

It is noteworthy that both contributions to the overall phase are suppressed by powers of $s / h$ : there is no phase if the bubble wall profile remains strictly along the $h$ direction. Bending of the path in the $s$ directions is necessary for generating any $\mathrm{CP}$ violation in the wall.

Although it might appear from (57) that one more integration is required to solve for $\theta(z)$, in fact we never need $\theta(z)$ itself, but only $\partial_{z} \theta$, to evaluate the CP-violating source term of the Boltzmann equations, to be discussed below. Eqn. (61) thus constitutes a sufficient solution for the phase of $m_{t}$ in the bubble wall.

\section{Numerical solution for bubble wall profiles}

The equations of motion can be solved numerically by shooting or by relaxation. However, for the purposes of doing an MCMC search, a simpler and faster approximate method is desirable. We first find the straight line in field space that connects the two VEVs and then locate, for each point on this line, the position along the plane perpendicular to the line where the potential is minimized. This deforms the line into a curve, which is the path of steepest descent through the saddle point. 

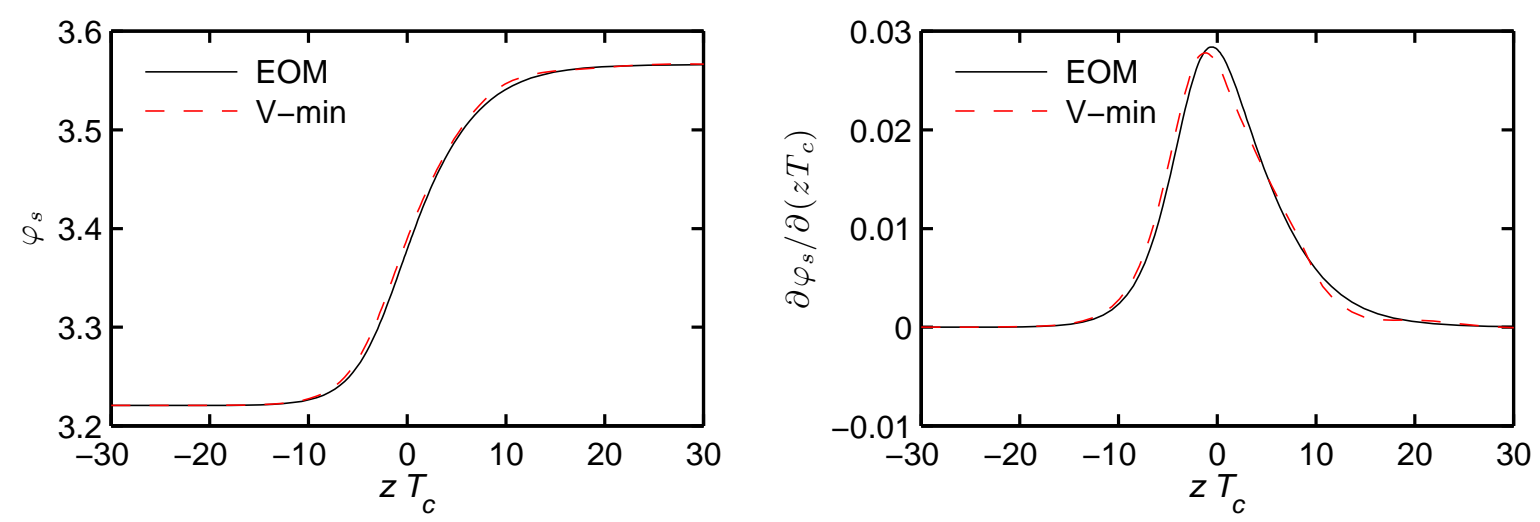

FIG. 5: Left: the phase of $S^{0}$-field computed from the full EOM (solid black) and from the minimization of $V$ (red dashed) as a function of $z T$ corresponding to model 1 in table IV Right: same for the $z T$ derivative of the phase.

One then solves the constrained one-dimensional problem along this path in the field space to determine the profile. This procedure is valid if the path does not curve very much (which we typically find to be true), but it tends to allow the path to curve more than the actual solution, since it ignores gradients of the fields when it determines the path. The real solution is systematically smoother than than the approximate one, which tends to overestimate the baryon asymmetry by a factor of $\lesssim 2$. The approximate method is thus sufficient for searching good candidates in our MCMC-runs, but for accurate results we recompute profiles for all models that pass the constraints by solving the equations of motion by use of accurate relaxation methods.

In Fig. 5 we show the solutions for the phase $\varphi_{s h}$ (left panel) and it derivative (right panel) as a function of $z T_{c}$ using the minimization of the potential (dashed curves) and the full equations of motion (solid curves) for the second model listed in table IV As claimed, the true solution is smoother than the one determined by constrained minimization of the potential. In Fig. 6 we display the profiles $\left\{h, s_{R}, s_{I}\right\}$ as a function of $z T_{c}$ for some other typical models. Based on these figures the wall thickness appears to be of order 10/T. This is an important parameter for electroweak baryogenesis and although we do not approximate the wall profiles by any analytic ansatz, it is convenient to characterize the wall thickness $L_{w}$ by fitting the total VEV $v(z)$ to the form $\frac{1}{2} v_{c} \tanh \left(z / L_{w}\right)$. In this way we find that $L_{w} T_{c} \sim 6-12$ for most models in our chains. The distribution of $L_{w}$ values is shown in Fig. 7 

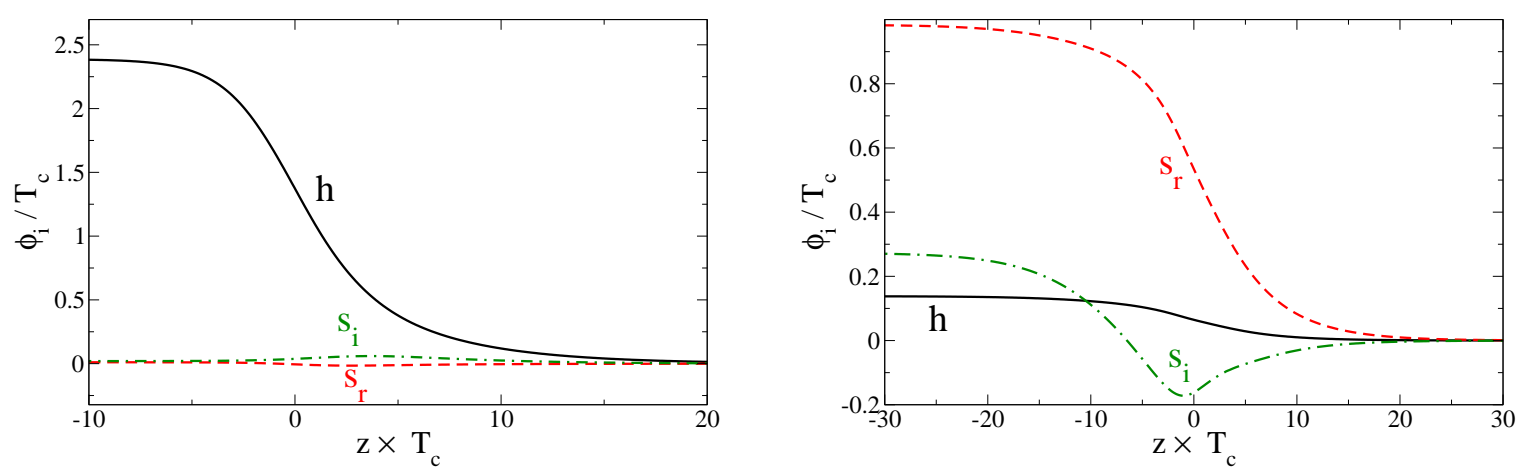

FIG. 6: Examples of bubble wall profiles for the fields $h, s_{R}, s_{I}$. Left: a typical profile; right: a profile leading to an atypically large CP-violating phase $\theta(z)$, and using model parameters that are ruled out by $R_{b}$ and $b \rightarrow s \gamma$ constraints.

\section{Comparison with previous results}

We have noted that the CP-violating phase $\theta(z)$ of the top quark mass is suppressed by the ratio $s / h$ in the bubble wall, and that $s / h$ is typically small for models that are not ruled out by particle physics constraints (see Fig. 6). This is in contrast to the earlier analysis of ref. [20]. Contrary to this one and earlier papers, we are finding a systematic suppression in the magnitude of $\theta(z)$ that goes beyond the smallness of $\mathrm{CP}$-violating phases in the potential. Namely, since $\langle S\rangle=0$ at zero temperature, but $\theta(z)$ vanishes if $S$ were to remain zero throughout the bubble wall, any nonvanishing $\theta(z)$ comes about as a finite-temperature effect, i.e., $\langle S\rangle$ being no longer zero in the broken minimum at finite $T$. On the other hand, ref. [20] did not observe any such suppression. The reason is that ref. [20] did not actually solve for the bubble wall profiles, but made the assumption that the two Higgs fields remained equal to each other in the wall, $\left|H_{1}\right|=\left|H_{2}\right|$ (which would translate into $|S|=0$ in our field basis). This assumption is only true if $H_{1} \leftrightarrow H_{2}$ is a symmetry of the Lagrangian, which ref. [20] claimed to be the case due to a choice of relations between the parameters of the Higgs potential. It would require $\lambda_{4}=\lambda_{5}=0$ in our language. But it is clear in that case that $|S|$ remains strictly zero in the bubble wall, so that $\theta(z)=0$. In the language of ref. [20], the CP-violating phases in the Higgs potential should vanish in order to have the symmetry $H_{1} \leftrightarrow H_{2}$ leading to $\left|H_{1}\right|=\left|H_{2}\right|$ in the wall, but this would also have made $\theta(z)=0$. In short, ref. [20] made inconsistent assumptions which obscured the suppression of $\theta(z)$ that we have made explicit. 


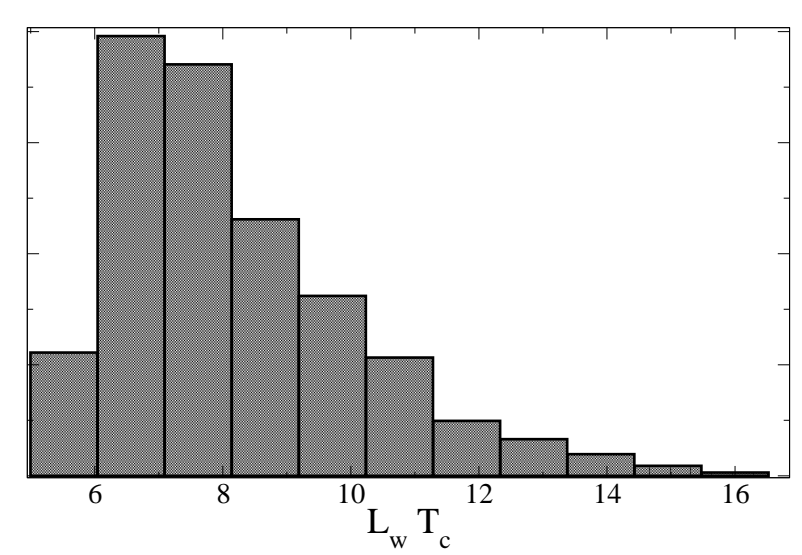

FIG. 7: Distribution of bubble wall thicknesses $L_{w}$, in units of $T_{c}^{-1}$

\section{B. Transport equations and source term}

The next step in determining the baryon asymmetry is to solve for the chemical potentials of relevant particle species in the vicinity of the bubble wall, that are induced by the top quark mass phase $\theta(z)$. One approach to deriving these equations is to start with the Boltzmann equations and to perturb the particle distribution for species $i$ away from its equilibrium form by allowing for a small chemical potential $\mu_{i}$ and a perturbation $\delta f_{i}$ that describes the departure from kinetic equilibrium [47]:

$$
f_{i}=\frac{1}{\exp \left(-\beta \gamma_{w}\left(E+v_{w} p_{z}\right)-\mu_{i}\right) \pm 1}+\delta f_{i}
$$

where $v_{w}$ is the bubble wall velocity and $\gamma_{w}=\left(1-v_{w}^{2}\right)^{-1 / 2}$. Linearizing in $\mu_{i}$ and $u_{i}=\left\langle\delta f_{i} v_{z}\right\rangle$, the perturbation to the particle's fluid velocity, leads to a set of coupled first order transport equations

for $\mu_{i}$ and $u_{i}$ [47]. This procedure has been carried out for the 2HDM in refs. [20, 48] and we adopt the same transport equations as in [20] which were tailored to two Higgs doublet models. Here the $\mathrm{CP}$-violating phase of $m_{t}$ provides the source term and the species that are followed are the quarks $t_{L}, t_{R}, b_{L}$ and the Higgs bosons, which are assumed to have a common chemical potential $\mu_{h}$ and velocity perturbation $u_{h}$.

The transport equations depend upon the bubble wall velocity $v_{w}$, which we take to be an undetermined parameter. In the computations that follow, we have chosen $v_{w}=0.1$ for definiteness, but we have checked that the final results for the baryon asymmetry do not depend strongly on this choice over a range of reasonable values. In fact the most important $v_{w}$-dependence is that which multiplies the source term, which we discuss next. However, in the end this dependence gets canceled by an explicit factor of $1 / v_{w}$ in the relation between the chemical potentials and 
the baryon asymmetry, eq. (66) below. The residual dependence of the result on $v_{w}$ comes only through its appearance in subleading terms in the transport equations, which explains the relatively weak effect. For example we find that the baryon asymmetry increases by only $20 \%$ when taking $v_{w} \rightarrow$ 0.05. (Ref. [14] which uses a different form for the diffusion equations finds a stronger dependence.)

\section{The source term}

A crucial ingredient in the transport equations is the inhomogeneous source term, which after many years is still a matter of controversy in the EWBG community. The source term adopted by [20] and by us encodes the fact that particles, and here the top quarks in particular, experience a CP-violating force while traversing the bubble wall, where $\theta(z)$ is varying [47, 49-52]. The corresponding force term in the Boltzmann equations thus gives rise to the (scaled dimensionless) source

$$
S_{t}=v_{w}\left(K_{8}\left(x_{t}\right)\left(x_{t}^{2} \theta^{\prime}\right)^{\prime}-K_{9}\left(x_{t}\right) x_{t}^{2} x_{t}^{2 \prime} \theta^{\prime}\right)
$$

where primes denote $\partial_{z T}$ and $K_{8,9}$ are dimensionless functions of $x_{t} \equiv\left|m_{t}\right| / T$ arising from phasespace averaging of certain kinematic variables, ${ }^{12}$ plotted in Fig. 8 ,

Since the typical wall thickness is significantly larger than $T_{c}^{-1}$, the derivatives in $S_{t}$ are a source of suppression of the baryon asymmetry. A source term with fewer derivatives could possibly give larger results. Such contributions arise from corrections due to $\mathrm{CP}$-violating dispersion relations to collision integrals in the Boltzmann equations. For example the Higgs decay to $t \bar{t}$ pairs contributes a source [53]:

$$
S_{\phi}=-v_{w} \frac{y_{t}^{2}}{8 \pi^{3}} I_{\phi} \Theta\left(M_{H}-2\left|m_{t}\right|\right) x_{t}^{2} \theta^{\prime}
$$

where $M_{H}$ is the mass of a Higgs boson, $\Theta$ is the Heaviside function and $I_{\phi}$ is a thermal kinematic function of $\left(M_{H} / T,\left|m_{t}\right| / T\right)$ typically of order $\sim 0.1$. However because of its small coefficient we find that the contribution of $S_{\phi}$ to the baryon asymmetry is always at least 10 times smaller than that of $S_{t}$.

The controversy alluded to above stems from the fact that there are competing formalisms for deriving source terms for the transport equations, that originate from refs. [54, 55]. These attempt to capture the quantum mechanical aspects of the particle interactions with the wall, rather than

\footnotetext{
$\overline{12}^{12}$ Our $K_{8,9}$ are related to those of [20] by simple $T$ scalings that make them dimensionless.
} 

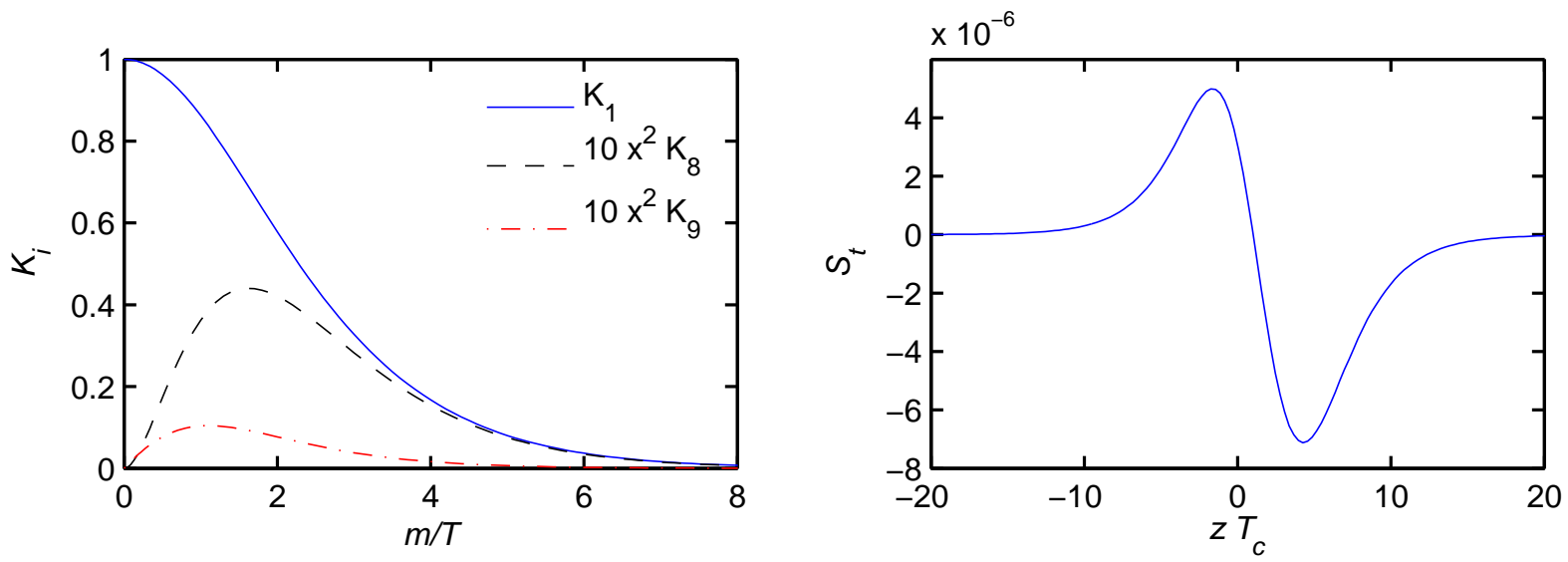

FIG. 8: Left: thermal functions $K_{8,9}$ appearing in the top quark source term, Eqn. 63), and $K_{1}$ which appears in the $B_{L}$ chemical potential, Eqn. (65). Right: an example (model 1 in table IV] of the source term as a function of $z T$. In this case the baryon asymmetry computed from full and 1D approximation to the field equations agree to $1 \%$.

the semiclassical behavior described by the sources (63)64). A difficulty with this approach is that rather drastic approximations need to be made in order obtain concrete, tractable expressions, notably expanding propagators to leading order in VEVs of the spatially varying Higgs fields (the "VEV insertion approximation"). The resulting source term has only a single derivative, unlike the semiclassical force source term we have adopted. It thus gives a larger estimate for the baryon asymmetry. However we do not believe that it represents a controlled approximation, so we adhere to the semiclassical approach. A more complete transport formalism, capable of accounting for the nonlocal coherence effects associated with the quantum interactions, is being developed in refs. [56]. There has been recent progress in improving upon the VEV insertion approach in [57, 58], although so far only with source involving scalar fields.

\section{Transport equations}

To solve the transport equations of ref. [20] accurately, it is necessary to use the relaxation method, since the asymptotic behavior of the solutions in the large $|z|$ regions cannot be determined analytically. On the other hand, shooting from large values of $\pm|z|$ toward $z=0$ is computationally much more efficient. The problem with shooting is that in principle one needs to know the analytic behavior of the asymptotic solutions in order to correctly specify the ratios $\mu_{i} / u_{i}$, 

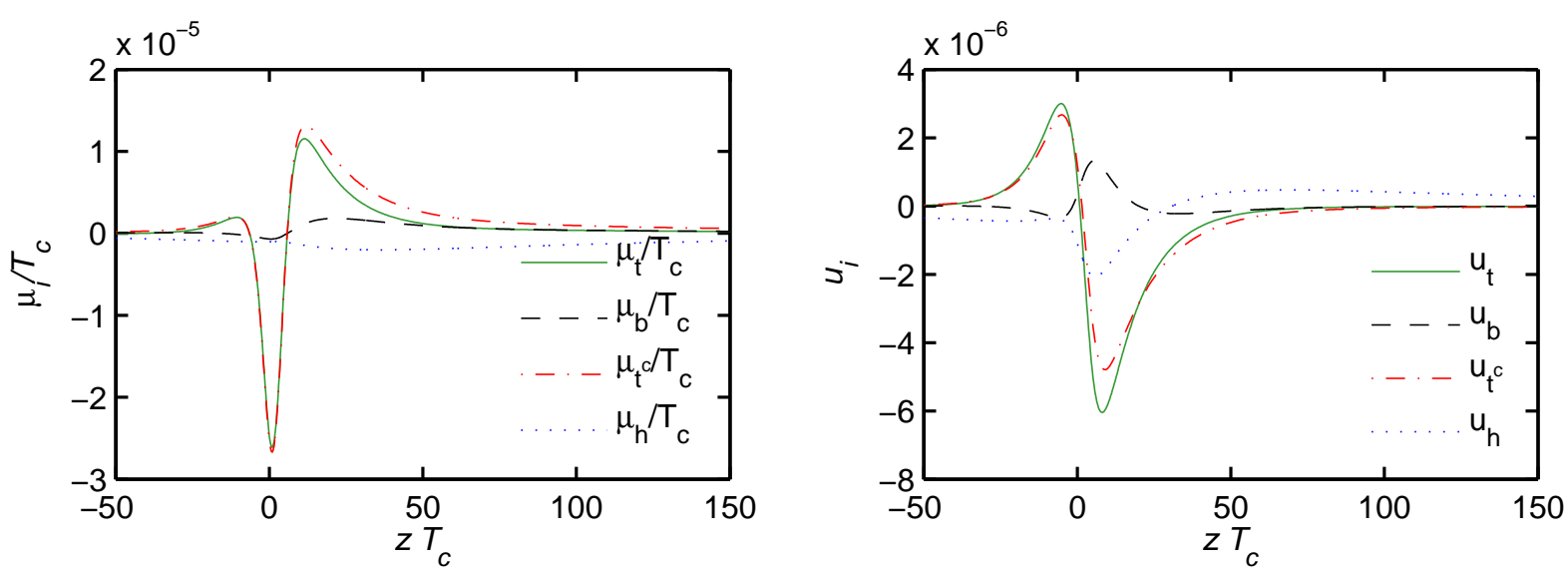

FIG. 9: Typical example of solution to transport equations for the chemical potentials of $t_{L}, t_{L}^{c}, b_{L}$ and Higgs bosons. This example (model 1 from table IV] gives a baryon asymmetry which is 1.1 times that of the observed value.

since only half of the independent variables can be input as free parameters (to be determined by smoothly matching the solutions at $z=0$ ) at either boundary. For example, one could freely vary $u_{i}$ at each boundary while holding $\mu_{i}$ fixed to find a solution, but one does not know beforehand what values of $\mu_{i}$ to choose. If the starting points are sufficiently far from $z=0$, then it might be a good approximation to take $\mu_{i}=0$ at the boundaries. But since the solutions are exponentially decaying functions of $|z|$, shooting is not numerically stable if the boundaries are taken to be so distant that setting $\mu_{i}=0$ would be a good approximation.

Of course the extra computational burden of relaxation is not an issue if one only needs to solve the transport equations for a small number of models, but to check thousands of models generated by the MCMC, a compromise is needed. We find that shooting typically gives an estimate for the baryon asymmetry that is accurate to within a factor of a few. We reserve relaxation for refining such estimates for potentially interesting examples. Typical examples of solutions for $\mu_{i}(z) / T_{c}$ and $u_{i}(z)$ from the relaxation method calculation are shown in Fig. 9.

\section{Baryon asymmetry results}

Once the chemical potentials around the bubble wall are known, they determine the overall asymmetry in left-handed baryon number, $\mu_{B_{L}}$, which biases the sphaleron interactions. Following 
[20], we take this to be

$$
\mu_{B_{L}}=\frac{1}{2}\left(1+4 K_{1, t}\right) \mu_{t}+\frac{1}{2}\left(1+4 K_{1, b}\right) \mu_{b}-2 K_{1, t} \mu_{t^{c}}
$$

where $K_{1, i}$ is a dimensionless function of $m_{i} / T$ shown in Fig. 8 . (Notice that $K_{1, b} \cong 1$ since $b$ is nearly massless.) The baryon-to-entropy ratio can then be calculated as

$$
\eta_{B}=\frac{405 \Gamma_{\mathrm{sph}}}{4 \pi^{2} v_{w} g_{*} T} \int d z \mu_{B_{L}} f_{\mathrm{sph}} e^{-45 \Gamma_{\mathrm{sph}}|z| /\left(4 v_{w}\right)}
$$

where $\Gamma_{\mathrm{sph}} \approx 10^{-6} T_{c}$ is the rate of sphaleron interactions in the symmetric phase, the exponential factor takes care of washout in the symmetric phase in case $v_{w} \ll 1$, and $f_{\text {sph }}$ takes into account the $z$-dependence of the actual sphaleron rate as it turns off in the broken phase. We estimate $f_{\text {sph }}$ as follows: in the broken phase where the Higgs VEV $v=\sqrt{2\left(\left|H^{0}\right|^{2}+\left|S^{0}\right|^{2}\right)}$ is nonzero, the sphaleron rate is given by ${ }^{13} \Gamma_{\mathrm{bp}} \sim N e^{-40 v / T}$. We fix $N$ by equating $\Gamma_{\mathrm{bp}} \equiv H_{c}^{-1}$ at $v / T_{c}=1$ (this encodes the usual sphaleron wash-out constraint $\left.v / T_{c}>1\right)$, giving $\Gamma_{\mathrm{bp}}=H_{c}^{-1} e^{40\left(1-v / T_{c}\right)} \approx$ $2.4 T_{c} e^{-40 v / T_{c}}$, where we used $H_{c} \approx 10^{-17} T_{c}$. We now assume that this equation holds also for $v=v(z)$ within the wall, and define the overall sphaleron rate to be $\min \left(\Gamma_{\mathrm{sph}}, \Gamma_{\mathrm{bp}}(z)\right) \equiv$ $\Gamma_{\mathrm{sph}} f_{\mathrm{sph}}(z)$ which gives

$$
f_{\mathrm{sph}}(z)=\min \left(1, \frac{2.4 T}{\Gamma_{\mathrm{sph}}} e^{-40 v(z) / T}\right) .
$$

It is important to use a realistic estimate for $f_{\mathrm{sph}}$ rather than a simple step function, because we find that the integral of $\mu_{B_{L}}$ over the symmetric phase can be much smaller than that of $\mu_{B_{L}} f_{\mathrm{sph}}$. This method also treats correctly those cases (and automatically cuts out the most extreme) where the VEV is nonzero even in the symmetric phase. The effect of the $f_{\mathrm{sph}}$ modulation is illustrated in Fig. 10.

We have searched the parameter space for models likely to provide a large enough baryon asymmetry and, optionally, a large enough dimuon excess in addition. We consider two cases. In the first, we set $\zeta_{b}^{\prime 0}=\zeta_{b}^{\prime \pm}=0$ so that the constraints from $R_{b}, b \rightarrow s \gamma$ and the neutron EDM are relaxed as much as possible without having to fine-tune the parameter $f$ in eq. (24). In this case we have or course given up on trying to explain the dimuon excess. We find that it is difficult to achieve a large enough baryon asymmetry even without this extra demand. A chain of 10,000 models designed to achieve large $v_{c} / T_{c}$ and $\theta(z)$ yields no models that have $\eta_{B}$ significantly greater

\footnotetext{
13 To be more precise the factor 40 in the exponent should be replaced by a function depending on the couplings in the scalar sector, but that would not change our results essentially.
} 


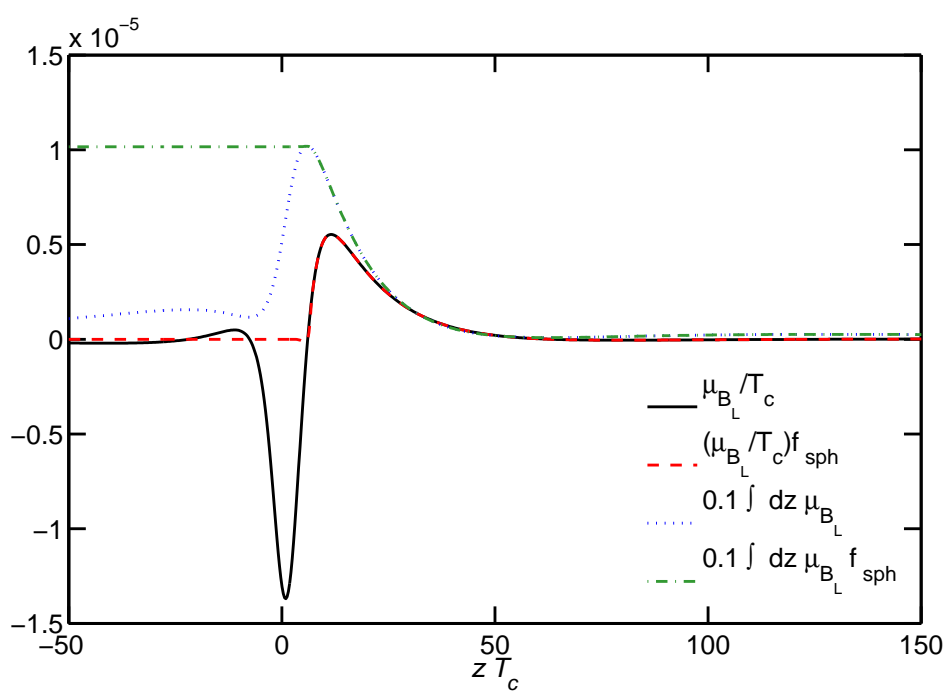

FIG. 10: Example of $z$-dependence of $\mu_{B_{L}}, \mu_{B_{L}} f_{\mathrm{sph}}$ and their integrals, showing the importance of the sphaleron rate modulation factor $f_{\mathrm{sph}}$. Symmetric phase is on the right.

than the observed value, and only a handfull which come close to the observed value. Rarely we find examples where the Landau pole occurs above the scale $\Lambda=3 \mathrm{TeV}$; working models with $\Lambda \cong 1 \mathrm{TeV}$ are much more frequent. The distribution of values of $\eta_{B}$ for these 10,000 models is shown in Fig. 11. It is remarkable that the upper limit on the baryon asymmetry happens to be so close to the observed value. In the figure we also show the distribution of $\eta_{B}$ in a chain where the constraints from EWPD, $R_{b}, b \rightarrow s \gamma$ and the neutron EDM are omitted; in that case it is easier to reproduce the observed baryon asymmetry, although there is still relatively little room for producing too great an asymmetry. Parameters for a few working models are given in table [II. They share several properties: light SM-like Higgs masses $\sim 117-125 \mathrm{GeV}$, new neutral and charged Higgs masses near $300 \mathrm{GeV}$, and values of $\left|\zeta_{t}^{0}\right| \sim 0.5$ that saturate the constraint coming from $R_{b}$, with $\zeta_{t}^{0}$ having a large phase $\theta$.

In the second case, we bias the Monte Carlo to produce models with large values of $\left|\zeta_{b}^{\prime 0}\right|$ to try to simultaneously explain the dimuon excess. In this scenario, while it is more difficult to achieve a large enough baryon asymmetry than in the previous one, the results are rather sensitive to the choice of $f$ in (24), with $f \rightarrow 0$ giving the most relaxed constraints. Similarly to the previous case, with a Landau pole cutoff of $2 \mathrm{TeV}$, we find no viable examples but at $1 \mathrm{TeV}$ and we find several (out of $10^{4}$ models in the chain) that have a large enough baryon asymmetry. If $f=1$, the actual values of $\eta_{D}^{\prime} \sim 1-2.5$ fall below the central values 3.6-4.6 needed to agree with the 


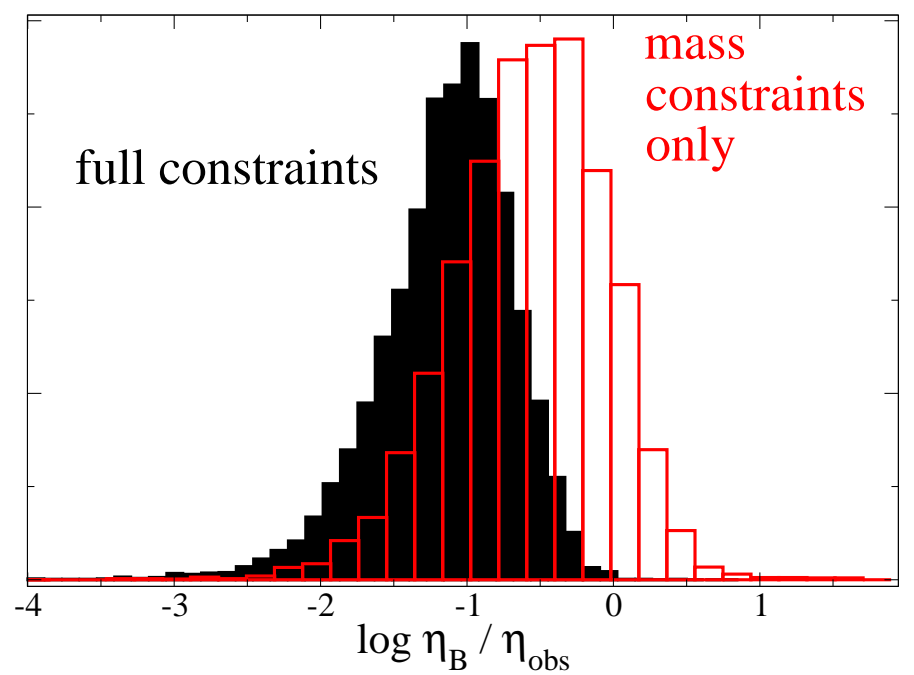

FIG. 11: Solid bars: frequency of $\eta_{B}$ values from chain of $10^{4}$ models that satisfy all constraints. Open (red) bars: frequency of $\eta_{B}$ when the $R_{b}, b \rightarrow s \gamma$ neutron EDM and perturbativity constraints are omitted.

\begin{tabular}{|c|c|c|c|c|c|c|c|c|c|c|c|c|c|c|c|}
\hline$m_{h}$ & $m_{1}$ & $m_{H}$ & $m_{A}$ & $m_{ \pm}$ & $\lambda_{1}$ & $\lambda_{2}$ & $\lambda_{3}$ & $\lambda_{4}^{R}$ & $\lambda_{4}^{I}$ & $\lambda_{6}$ & $\left|\eta_{U}\right|$ & $\theta$ & $\frac{v_{c}}{T_{c}}$ & $\Lambda$ & $\frac{\eta_{B}}{\eta_{\text {obs }}}$ \\
\hline 117 & 77 & 289 & 327 & 299 & 2.96 & 0.19 & -0.20 & -0.56 & -0.17 & 0.04 & 0.42 & -0.97 & 1.50 & 3304 & 1.00 \\
121 & 141 & 300 & 407 & 307 & 2.46 & 1.12 & -0.63 & 0.10 & 0.25 & 1.96 & 0.52 & 3.83 & 1.10 & 1019 & 1.32 \\
125 & 121 & 374 & 325 & 310 & 2.68 & 0.89 & 0.28 & -0.22 & -0.27 & 1.66 & 0.52 & 3.41 & 1.07 & 1146 & 1.20 \\
123 & 129 & 263 & 399 & 292 & 2.26 & 0.97 & -0.74 & 0.12 & 0.22 & 2.14 & 0.43 & 3.68 & 1.04 & 1007 & 1.18 \\
\hline
\end{tabular}

TABLE III: Parameters of sample models with $\zeta_{b}^{\prime 0}=\zeta_{b}^{\prime \pm}=0$ (hence $\eta_{D}^{\prime}=0$, and no dimuon anomaly) and sufficient baryogenesis, and a Landau pole at scale $\Lambda$. Masses/energies are in GeV.

$\mathrm{D} \emptyset$ dimuon observation. However a modest relaxation of the constraints by using $f=0.5$ leads to more favorable results, where we find $\sim 10$ models with the desired baryon asymmetry and dimuon signal. (The distribution of models satisfying both constraints is displayed graphically in the final figure in section VIII) These examples are similar to those with $\zeta_{b}^{\prime 0}=\zeta_{b}^{\prime \pm} \approx 0$ in that the SM-like Higgs must be light, while the new scalars exceed $300 \mathrm{GeV}$ in mass.

\section{EXPERIMENTAL TESTABILITY}

We have shown that a direct link between the new phase hinted at in $B$ physics data and EWBG cannot be made in 2HDM models in general. EWBG depends upon CP violation in the scalar 


\begin{tabular}{|c|c|c|c|c|c|c|c|c|c|c|c|c|c|c|c|c|c|}
\hline$m_{h}$ & $m_{1}$ & $m_{H}$ & $m_{A}$ & $m_{ \pm}$ & $\lambda_{1}$ & $\lambda_{2}$ & $\lambda_{3}$ & $\lambda_{4}^{R}$ & $\lambda_{4}^{I}$ & $\lambda_{6}$ & $\left|\eta_{U}\right|$ & $\theta$ & $\left|\eta_{D}^{\prime}\right|$ & $\left|\eta_{\mathrm{D} \phi}^{\prime}\right|$ & $\frac{v_{c}}{T_{c}}$ & $\Lambda$ & $\frac{\eta_{B}}{\eta_{\mathrm{obs}}}$ \\
\hline 121 & 212 & 350 & 523 & 355 & 2.67 & 2.39 & -1.25 & 0.630 & -0.545 & 0.08 & 0.560 & -2.25 & 4.79 & 4.4 & 1.65 & 1675 & 1.14 \\
117 & 168 & 276 & 453 & 316 & 2.36 & 1.35 & -1.06 & 0.052 & -0.215 & 1.40 & 0.381 & -2.00 & 5.95 & 3.3 & 1.24 & 1382 & 1.04 \\
119 & 199 & 301 & 485 & 318 & 2.03 & 2.06 & -1.20 & 0.046 & -0.168 & 1.73 & 0.463 & -2.23 & 4.41 & 3.7 & 1.22 & 1160 & 1.22 \\
\hline
\end{tabular}

TABLE IV: Sample of model parameters giving sufficiently large $\left|\eta_{D}^{\prime}\right|$ (hence $\left.\zeta_{b}^{\prime 0}\right)$ and sufficient baryogenesis. $\eta_{\mathrm{D} \emptyset}^{\prime}$ is the value needed to match the central value of the dimuon anomaly.

potential and top quark Yukawa couplings, while enhanced $B_{s}$ mixing involves new couplings to the $b$ quark and phenomenological constraints severely limit the latter couplings. Nevertheless, the prospects for direct collider confirmation (or refutation) of the new phase hinted at in the dimuon data and the new scalar degrees of freedom we have discussed are very strong. In this section we briefly discuss some of these prospects.

\section{A. Confirmation/refutation of the dimuon anomaly}

The hint for new physics near the electroweak scale indicated by the $\mathrm{D} \phi$ collaboration [2] that has at least one new source of $\mathrm{CP}$ violation, is consistent with other direct measurements that are sensitive to the same phase in $B_{s}$ mixing and use flavour tagged $B_{s}^{0} \rightarrow J / \Psi \phi$ decays. With $6.1 \mathrm{fb}^{-1}$ of data, $\mathrm{D} \varnothing$ [59] finds a hint of a new phase in decays of this form; their results show a larger deviation from the SM than the equivalent measurements by CDF [60]. Both results are statistically consistent. A more recent measurement that has appeared since the original version of this work includes $8 \mathrm{fb}^{-1}$ of data from D $\varnothing$ reported in [3]. A method for checking the consistency of these results can be found in [21, 61]: one can compare the fit values of $\Delta \Gamma_{s}$ and $S_{\phi \psi}$ from the two measurements. The relevant relation is

$$
\left|\Delta \Gamma_{s}\right| \simeq-\left[0.20 \pm 0.11 \mathrm{ps}^{-1}\right] \sqrt{1-S_{\phi \psi}^{2}} / S_{\phi \psi}
$$

using $\delta m_{s}=17.78 \pm 0.12 \mathrm{ps}^{-1}, a_{S L}^{d}=-(4.7 \pm 4.6) \times 10^{-3}$ and $a_{S L}^{b}=-(0.787 \pm 0.172 \pm$ $0.093) \times 10^{-2}$. Using $S_{\phi \psi}=-0.55 \pm 0.35$ one finds that [3] implies

$$
\frac{\left|\Delta \Gamma_{s}\right| S_{\phi \psi}}{\sqrt{1-S_{\phi \psi}^{2}}} \simeq-0.11 \pm 0.10 \mathrm{ps}^{-1}
$$


which is consistent with the updated value derived from the dimuon anomaly. It is encouraging that $\mathrm{D} \varnothing$ has just announced that with $9 \mathrm{fb}^{-1}$ of data the significance of this deviation in the dimuon measurement is now $3.9 \sigma$ compared directly against the SM prediction of the dimuon charge asymmetry.

The first preliminary measurements of [62] flavour tagged $B_{s}^{0} \rightarrow J / \Psi \phi$ decays at $\mathrm{LHC}_{\mathrm{b}}$ using $36 \mathrm{pb}^{-1}$ of data found a (statistics limited) result that agreed well with the past measurements of D $\varnothing[59]$ and CDF [60], and was consistent with the dimuon anomaly. However a more precise measurement was subsequently reported at Lepton-Photon 2011 that introduces significant tension into the interpretation of the pattern of data as implying new physics. This measurement reports $\Delta \Gamma_{s} \simeq 0.123 \pm 0.029 \pm 0.008$ and $S_{\phi \psi}=0.13 \pm 0.18 \pm 0.07$, which gives

$$
\frac{\left|\Delta \Gamma_{s}\right| S_{\phi \psi}}{\sqrt{1-S_{\phi \psi}^{2}}} \simeq 0.021 \pm 0.024 \mathrm{ps}^{-1} .
$$

These results are to be compared to the result derived from the updated dimuon anomaly data $-0.20 \pm 0.11 \mathrm{ps}^{-1}-$ demonstrating a tension at about the $2 \sigma$ level.

This new $\mathrm{LHC}_{\mathrm{b}}$ result is not a direct measurement of the dimuon asymmetry, but the prospect for such a determination is promising. In part due to the improved mass resolution at $\mathrm{LHC}_{\mathrm{b}}$, a measurement of the difference in the asymmetry in $B_{s}$ mixing $\left(a_{f s}^{s}\right)$ and $B_{d}$ mixing $\left(a_{f s}^{d}\right)$ is being pursued. This approach has the added benefit of detector asymmetries (but not production asymmetries) cancelling through taking the difference. For both $a_{f s}^{s}$ and $a_{f s}^{d}$, semileptonic $B \rightarrow$ $D \mu^{-} \bar{\nu}$ decays and subsequent kaon-rich decays of $D_{s}^{+} \rightarrow K^{+} K^{-} \pi^{+}$and $D^{+} \rightarrow K^{+} K^{-} \pi^{+}$are used for event selection. Measuring $a_{f s}^{s}-a_{f s}^{d}$ also has the added benefit of producing an orthogonal constraint in the $a_{f s}^{s}, a_{f s}^{d}$ plane which offers strong prospects of direct confirmation or refutation of the dimuon anomaly in the 2011 data set, if limitations from the production asymmetries can be overcome.

At this time, available combined fits indicate a $3.3 \sigma$ deviation from the SM [4, 21] based on the older measurement and not incorporating the new $\mathrm{LHC}_{\mathrm{b}}$ and $\mathrm{D} \emptyset$ measurements. We use this result in our analysis. In the model we have studied and attempted to link to baryogenesis, one expects $h_{s}=h_{d}$ and $\sigma_{s}=\sigma_{d}$, and the best fit values are $h_{q}=0.255$ and $2 \sigma_{q}=180^{\circ}+63.4^{\circ}$ based on the smaller data set. 


\section{B. Collider prospects for 2HDMs with successful EWBG}

From a collider search perspective, the highly constrained mass spectrum and parameter space consistent with EWBG is a positive feature, since the predictions for collider signatures are sharpened. The typical mass scales of the new scalar states with successful baryogenesis fall into two categories. When we set $\zeta_{b}^{0}=\zeta_{b}^{\prime \pm} \approx 0$ in our scans we find typical values $260 \lesssim m_{H} \lesssim 360 \mathrm{GeV}$, $350 \lesssim m_{A} \lesssim 550 \mathrm{GeV}$ and $300 \lesssim m_{ \pm} \lesssim 350 \mathrm{GeV}$ as is shown in fig [3. Alternatively, if we bias the MC search to give as large a value of $\zeta_{b}^{0}=\zeta_{b}^{\prime \pm}$ as possible then we find typical masses $275 \lesssim m_{H} \lesssim 375 \mathrm{GeV}, 475 \lesssim m_{A} \lesssim 575 \mathrm{GeV}$ and $400 \lesssim m_{ \pm} \lesssim 500 \mathrm{GeV}$. In both cases, the simultaneous best fit value for the Higgs mass is tightly bounded around $115 \lesssim m_{H} \lesssim 130 \mathrm{GeV}$. Further, in both cases $\lambda_{5} \sim 0$ for successful EWGB, so the new scalars, when produced, do not dominantly decay to pairs of Higgs particles and the mass and VEV eigenstates of the new scalars approximately coincide. This makes discovery of these states extremely challenging when the scalar masses are above the $t \bar{t}$ mass threshold as we will show. SM Higgs collider phenomenology is not significantly affected in this scenario, and we focus our discussion on the possible collider signatures of $m_{H}, m_{A}, m_{ \pm}$. The challenges of discovering charged scalars at LHC are well known and are not alleviated in this model, so we focus our discussion on the neutral scalars.

\section{Tevatron Physics}

The lower end of the above mass intervals are well within the kinematic reach of the Tevatron and the simplest topology of interest at colliders is a resonant $s$-channel tree-level exchange of the new scalar states. The partonic tree-level differential cross section for $m_{H}, m_{A}$ is given by

$$
\frac{d \hat{\sigma}}{d \hat{t}}=\frac{1}{192 \pi \hat{s}} \frac{1}{|\overrightarrow{\vec{p}}|^{2}} \frac{\left(s-m_{1}^{2}-m_{2}^{2}\right)\left(s-m_{3}^{2}-m_{4}^{2}\right)}{\left(s-m_{H / A}^{2}\right)^{2}+\Gamma_{S_{R} / S_{I}}^{2} m_{H / A}^{2}}\left|f_{i j}^{U / D}\right|^{2}\left|f_{k l}^{U / D}\right|^{2} .
$$

We denote partonic variables with hat superscripts and the masses are labeled with 1,2 for initial states and 3,4 for final states. Here the three-momenta of the initial SM states in the center of mass (CM) frame are $\hat{\vec{p}}$. The general coupling of the scalar fields to initial state flavours $i, j$ and final state flavours $k, l$ is given by

$$
f_{i j}^{U / D}=y_{i}^{U / D}\left[\delta_{i, j} \eta_{U / D}+\eta_{U / D}^{\prime} V_{i n}\left(y_{n}^{U / D}\right)^{2} V_{n j}^{\dagger}+\delta_{i, j} \tilde{\eta}_{U / D}^{\prime}\left(y_{b}^{U / D}\right)^{2}+\cdots\right],
$$

where $y_{i}^{U / D}=\sqrt{2} m_{i}^{U / D} / v$. The expression for $f_{k l}$ is identical. Considering parton distribution function (PDF) and CKM suppression, the largest effect of these exchanges at the Tevatron is 
expected to be on the production of $t \bar{t}$ final states. Specializing to this case, neglecting all initial state masses in the kinematic expressions, and taking $y_{t} \sim 1$, we find the partonic cross section for $i, j$ initial states that must be convoluted with PDF's is given by

$$
\hat{\sigma}_{i j}=\frac{1}{192 \pi} \frac{\left(s-2 m_{t}^{2}\right)}{\left(s-m_{H / A}^{2}\right)^{2}+\Gamma_{S_{R} / S_{I}}^{2} m_{H / A}^{2}}\left|f_{i j}\right|^{2}\left(\zeta_{t}^{0}\right)^{2} .
$$

It is interesting that there is an excess in the lowest mass bin between 350 and $400 \mathrm{GeV}$ in the top quark invariant mass distribution. This range approximately coincides with the masses preferred for EWBG in our model when $\zeta_{b}^{\prime \pm} \approx 0$. However, we find that the maximum enhancement of the top quark invariant mass distribution in the relevant bin is too small by a factor of $\sim 100$ for couplings consistent with particle physics constraints and successful EWBG. The effects of treelevel exchanges of neutral scalars consistent with EWBG in other observables at the Tevatron are similarly too small to be observed with the current data set.

\section{LHC Physics}

The production of the neutral scalars through the process $g g \rightarrow s_{R / I}$ at the Tevatron and LHC is a promising channel for this scenario. When we bias for large values of $\zeta_{b}^{\prime 0}$ the process $p p \rightarrow s_{R} b \bar{b}$ at LHC is also promising[25]. Conversely, for the parameter sets consistent with EWBG we find that the production cross sections for $p \bar{p} \rightarrow W^{ \pm} \rightarrow s^{ \pm} s_{R / I}$ at the Tevatron and $p p \rightarrow$ $W^{ \pm} \rightarrow s^{ \pm} s_{R / I}$ at LHC are $\lesssim 10^{-3} \mathrm{fb}$. In Table $\nabla$ we display these cross sections for several 2HDMs consistent with EWGB: five sets in which $\zeta_{b}^{\prime \pm} \approx 0$, and five sets in which $\zeta_{b}^{\prime \pm}$, using the stronger particle physics constraints that arise from assuming that $f=1$ in the relation (24) between $\zeta_{b}^{\prime \pm}$ and $\zeta_{b}^{0}$. Table VI gives the corresponding results for models that can satisfy both the requirements of baryogenesis and the Tevatron dimuon anomaly, using the relaxed constraints from taking $f=0.5$.

For the parameter sets consistent with EWBG, we find that the production through gluon fusion indicates that LHC's current run (with $\gtrsim 1 \mathrm{fb}^{-1}$ on tape) should have produced hundreds of events. The branching ratios of the neutral scalars are determined using the results summarized in [63]. Recall our convention that the field $S$ does not get a VEV, because of this and the fact that $\left|\lambda_{5}\right| \ll 1$, the mass eigenstate fields only get small VEVs of the order

$$
\left\langle s_{R}^{\prime}\right\rangle=v \epsilon_{S_{R}}=\frac{v^{3} \lambda_{5}^{R}}{m_{H}^{2}-m_{h}^{2}}, \quad\left\langle s_{I}^{\prime}\right\rangle=v \epsilon_{S_{I}}=\frac{v^{3} \lambda_{5}^{I}}{m_{A}^{2}-m_{h}^{2}} .
$$




\begin{tabular}{|c|c|c|c|c|c|c|c|}
\hline \hline$m_{ \pm}$ & $m_{H}$ & $m_{A}$ & $\zeta_{t}^{0}$ & $\zeta_{b}^{\prime \pm}$ & $\sigma_{\left(g g \rightarrow s_{R}\right)}(\mathrm{fb})$ & $\sigma_{\left(g g \rightarrow S_{A}\right)}(\mathrm{fb})$ & $\sigma_{\left(p p \rightarrow s_{R} b \bar{b}\right)}(\mathrm{fb})$ \\
\hline 310 & 374 & 325 & -0.52 & 0 & 275 & 895 & - \\
317 & 332 & 337 & -0.45 & 0 & 192 & 762 & - \\
292 & 263 & 399 & 0.43 & 0 & 239 & 506 & - \\
307 & 300 & 407 & 0.52 & 0 & 283 & 662 & - \\
315 & 283 & 462 & -0.43 & 0 & 211 & 221 & - \\
\hline 474 & 370 & 559 & -0.52 & 0.62 & 277 & 105 & 0.8 \\
412 & 304 & 497 & 0.42 & 1.16 & 279 & 212 & 7.3 \\
395 & 286 & 480 & -0.43 & 1.18 & 304 & 260 & 9.8 \\
408 & 307 & 488 & -0.44 & 1.15 & 274 & 236 & 6.7 \\
435 & 311 & 532 & -0.51 & 0.93 & 270 & 141 & 4.2 \\
\hline \hline
\end{tabular}

TABLE V: Sample of production cross sections for models with successful EWGB, but insufficient dimuon excess. We use MSTW2008 PDF's in determining the cross sections and use the renormalization scale $\mu=m_{H / A} / 2$ for the PDF's. The cross sections are evaluated for LHC at an operating energy of $7 \mathrm{TeV}$ at leading order. We use the results of [63, 64] for the LO cross sections. Here we have assumed the generic relation (24) with $f=1$ between $\zeta_{b}^{\prime \pm}$ and $\zeta_{b}^{0}$.

\begin{tabular}{|c|c|c|c|c|c|c|c|c|}
\hline \hline$m_{ \pm}$ & $m_{H}$ & $m_{A}$ & $\zeta_{t}^{\prime 0}$ & $\zeta_{b}^{\prime 0}$ & $\zeta_{b}^{\prime 0} /\left(\zeta_{b}^{\prime 0}\right)_{\mathrm{D} \phi}$ & $\sigma_{\left(g g \rightarrow S_{H}\right)}(\mathrm{fb})$ & $\sigma_{\left(g g \rightarrow S_{A}\right)}(\mathrm{fb})$ & $\sigma_{\left(p p \rightarrow S_{H} b \bar{b}\right)}(\mathrm{fb})$ \\
\hline 355 & 350 & 523 & -0.56 & 4.8 & 1.08 & 312 & 182 & 16 \\
315 & 276 & 453 & -0.38 & 6.0 & 1.81 & 171 & 194 & 74 \\
388 & 352 & 554 & -0.51 & 5.9 & 1.37 & 262 & 107 & 24 \\
368 & 350 & 545 & -0.40 & 5.9 & 1.36 & 159 & 72 & 24 \\
336 & 303 & 498 & -0.40 & 5.7 & 1.30 & 165 & 124 & 44 \\
\hline \hline
\end{tabular}

TABLE VI: Same as table V, but now with sufficient dimuon excess, allowed by mild relaxation of the relationship (24) between $\zeta_{b}^{\prime 0}$ and $\zeta_{b}^{\prime}{ }^{ \pm}$from $f=1$ to $f=0.5$.

When EWBG occurs, these vacuum expectation values are $\left\langle s_{R}^{\prime}\right\rangle \simeq\left\langle s_{I}^{\prime}\right\rangle \simeq 10 \mathrm{GeV}$. This significantly suppresses decays to $W W, Z Z$ making these scalars difficult to discover at LHC. The 
largest decay widths are typically to fermions with

$$
\Gamma\left(s_{R / I} \rightarrow f \bar{f}\right)=\frac{G_{F} N_{C}}{8 \sqrt{2} \pi} v^{2}\left|\zeta_{f}^{0}\right|^{2} m_{H / A} \beta_{f}^{n},
$$

and $\beta=\left(1-4 m_{f}^{2} / m_{H / A}^{2}\right)^{1 / 2}$. Here $n=3$ for $m_{H}$ and $n=1$ for $m_{A}$. The neutral scalars decay almost exclusively into $t \bar{t}$ final states when they are above the $t \bar{t}$ threshold. Other phenomenologically interesting final states due to their clean collider signatures are $\gamma \gamma, Z Z, W W$ and $\tau^{+} \tau^{-}$. We show in Fig. (12) the dominant branching ratios as a function of the scalar masses for typical parameter values. We also show the branching ratios to EW final states that are potentially promising for discovery.
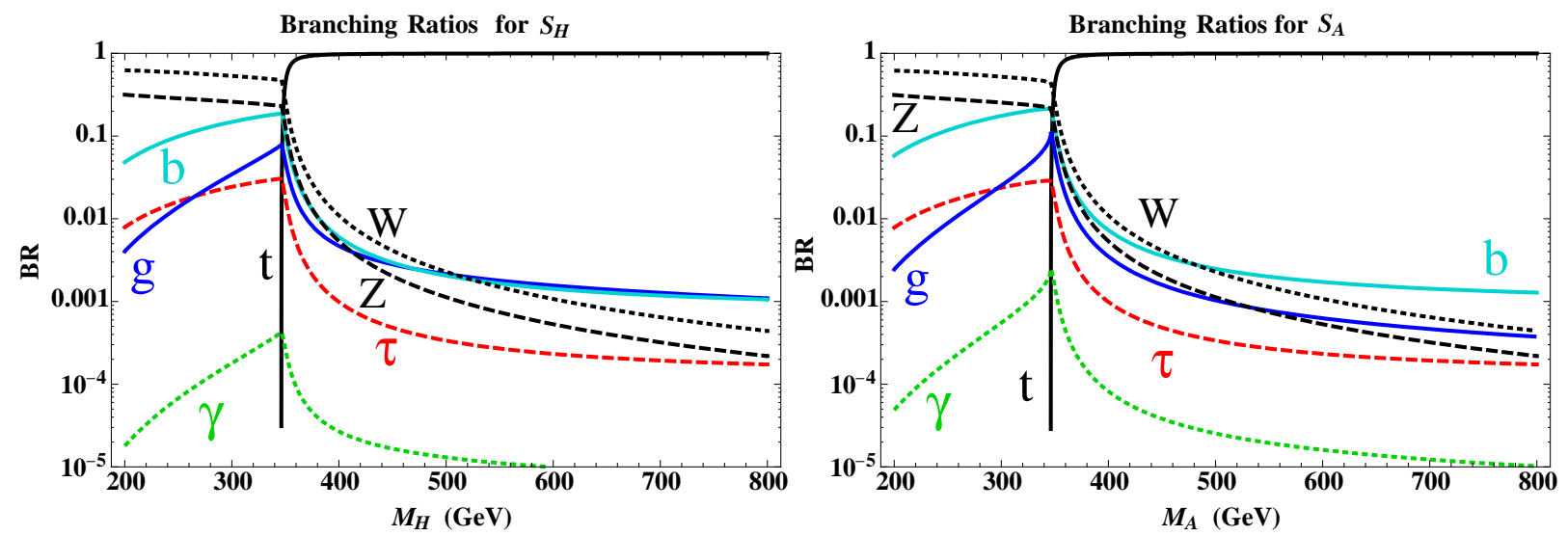

FIG. 12: Branching ratios for the heavy neutral scalars. The branching is almost exclusively to $W W$ and $Z Z$ below the top quark threshold and to $t \bar{t}$ above the top threshold. Here we have neglected flavourchanging decays and decays to $h h$, the later being highly suppressed compared to the leading decay to $W W$ and $Z Z$ or $t \bar{t}$ and challenging to discover. Each curve is labeled by the final state particle $X$ for the decay $s_{R / I} \rightarrow X \bar{X}$. We have taken $\zeta_{t}^{0}=0.5, m_{t}=173.1$ and $\zeta_{b}^{0}=2 m_{b}^{2} / v^{2}$. Flavour changing decays to $t \bar{c}$ or $t \bar{u}$ require an insertion of $2 m_{b}^{2} / v^{2}$ and further CKM suppression. They are given essentially by CKM suppressed rescalings of the shown $\operatorname{Br}(b \bar{b})$.

Below the $t \bar{t}$ threshold, strong discovery prospects exist through decays to $W W$ and $Z Z$ using standard searches. Typically in these scenarios at least one heavy neutral scalar is below the $t \bar{t}$ threshold. However above the $t \bar{t}$ threshold, discovery prospects are much dimmer. When $m_{H / A}>2 m_{t}$ large numbers of resonant $t \bar{t}$ pairs are produced. Unfortunately the $t \bar{t}$ production from QCD is far larger because the signal process is loop-suppressed. For example, for $s_{I} \rightarrow t \bar{t}$ with a mass scale of $m_{A} \sim 500 \mathrm{GeV}$, the approximate cross section for $\sigma\left(g g \rightarrow s_{I}\right)$ is $0.2 \mathrm{fb}$ 
while the QCD $t \bar{t}$ background with $M_{t \bar{t}} \sim 500 \mathrm{GeV}$ is given by $\sim 10 \mathrm{pb}$ at leading order. The first measurements of the $t \bar{t}$ invariant mass spectrum at LHC have recently appeared [65]. However it is unlikely that the resonant production of $t \bar{t}$ due to these heavy scalars can be uncovered in the $t \bar{t}$ invariant mass spectrum for quite some time. Precision studies would have to be performed, possibly using the angular distributions of the reconstructed $t \bar{t}$ pair since this signal is through a scalar resonance. After event selection cuts, the reconstructed partonic $t \bar{t}$ spectra has only 5 events in the $M_{t \bar{t}} \sim 500 \mathrm{GeV}$ mass region in this study. Thus total event selection cut suppression of the background and signal is $\sim 10^{-3}$.

Due to the difficulty of overcoming the $t \bar{t}$ QCD background, and the lack of efficient light flavour tagging at LHC to uncover, for example, CKM suppressed $t \bar{c}$ decays, utilizing a highly suppressed branching ratio such as to $\tau^{+} \tau^{-}$might be considered more hopeful. The dominant background $^{14}$ in this case is the inclusive process $p p \rightarrow Z X \rightarrow \tau^{+} \tau^{-} X$, whose tree-level electroweak contribution has an antiquark initial-state PDF suppression at LHC. The partonic cross section for this background is given by

$$
\hat{\sigma}_{f}=\frac{\hat{s}(4 \pi)^{2} \alpha_{E W}^{2}}{48 \pi \sin ^{4} \theta_{W} \cos ^{4} \theta_{W}} \frac{\left[\left(g_{V}^{f}\right)^{2}+\left(g_{A}^{f}\right)^{2}\right]\left[\left(g_{V}^{\tau}\right)^{2}+\left(g_{A}^{\tau}\right)^{2}\right]}{\left(\hat{s}-m_{z}^{2}\right)^{2}+m_{z}^{2} \Gamma_{Z}^{2}}
$$

Here $g_{V}, g_{A}$ are the usual electroweak couplings that depend on the isospin of the initial state fermions $f$. We use $\Gamma_{Z}=2.5 \mathrm{GeV}$ and $m_{z}=91.2 \mathrm{GeV}$. We use hat superscripts to denote partonic quantities. The expression we use to implement cuts on the $2 \rightarrow 2$ processes of interest, in $\mathrm{fb}$ units, is given by

$$
\frac{d \sigma}{d M_{\tau \tau}}=\frac{0.3894 \times 10^{12} M_{\tau \tau}}{s_{h a d}} \int_{-y_{B}^{0}}^{y_{B}^{0}} d y_{B} \int_{-z_{0}}^{z_{0}} d z \sum_{f} f_{f}\left(R e^{y_{B}}\right) f_{\bar{f}}\left(R e^{-y_{B}}\right) \hat{\sigma}_{f}
$$

where $R=\sqrt{M_{\tau \tau}^{2} / s_{h a d}}, f_{f}, f_{\bar{f}}$ are the 2008 MSTW parton densities of the initial state quarks, $s_{\text {had }}$ is the hadronic center of mass energy, and $Y_{B}$ is the boost rapidity of the subprocess frame. We treat the highly boosted $\tau$ 's as $\tau$ jets required to have rapidity $\left|y_{1,2}\right|<Y_{\max }$ with cuts on $p_{T}$ of $p_{T}^{\min }$ so that

$$
\begin{aligned}
y_{B}^{0} & =\operatorname{Min}\left[Y_{\max }-\log \left[M_{\tau \tau}^{2} / s\right] / 2\right] \\
z^{0} & =\operatorname{Min}\left[\sqrt{1-4\left(p_{T}^{\min }\right)^{2} / M_{\tau \tau}^{2}}, \tanh \left(Y_{\max }-\left|y_{B}\right|\right)\right]
\end{aligned}
$$

\footnotetext{
${ }^{14}$ This is most difficult to significantly reduce with selection cuts. A background of $t \bar{t}$ also exists with $\tau$ pairs produced in the decay of the tops. The latter should be more reducible due to its associated missing energy and multi-jet topology.
} 
This result for hadronic cuts on $2 \rightarrow 2$ processes is consistent with [66]. We find that the $d \sigma / d M_{\tau \tau}$ invariant mass spectrum has a peak of resonantly produced $\tau^{+} \tau^{-}$final states typically $10^{-3}$ the background spectrum when a $p_{T}$ cut of $10 \mathrm{GeV}$ is applied to the leptons and the $\tau$ jets are restricted to have a rapidity of $<3$. Thus discovery in this channel is unlikely. Above the $t \bar{t}$ threshold, relying on decays to $W W$ and $Z Z$ offers some prospects for discovery.

\section{DISCUSSION AND CONCLUSIONS}

In this paper we have given an exhaustive account of EWBG in two-Higgs doublet models. Our study, while focusing on MFV Yukawa couplings and a simultaneous explanation of the D $\varnothing$ dimuon excess, is general and encompasses earlier popular choices of Yukawa couplings, such as type I and II models. The MFV two Higgs doublet model also offers a controlled theoretical framework where new CP violating phases can appear at the EW scale without introducing inconsistencies with a multitude of other flavour measurements. Unlike previous works, we have considered the most general set of couplings in the Higgs potential and have made no simplifying assumptions to reduce the parameter space. Instead we used a Markov Chain Monte Carlo to explore the full space of models, aiming toward those with a strong first order phase transition and large $\mathrm{CP}$ violation in the bubble walls.

Despite this, we find it more difficult to obtain a large enough baryon asymmetry than previous studies indicated. This is partly because we have imposed more phenomenological constraints; in addition to accelerator mass bounds and the neutron EDM that were considered in previous studies, we included EWPD, $R_{b}, b \rightarrow s \gamma$ and perturbativity of couplings up to a minimum Landau pole scale of $1-2 \mathrm{TeV}$. In addition, we have avoided simplifying assumptions about the shape of the bubble wall profile that artificially increased the baryon asymmetry in previous work. For every model in the chain from the Monte Carlo, we numerically determined the actual solutions for the bubble wall profiles and the $\mathrm{CP}$-violating phase $\theta(z)$ that sources baryogenesis. In the process we elucidated a dynamical mechanism by which $\theta(z)$ tends to be suppressed: the existence of nonzero $\theta(z)$ depends upon exciting the field $S$ whose VEV is zero at $T=0$. Thus there is finite-temperature suppression factor inherent in $\theta(z)$ which would be overlooked in a seemingly reasonable guess for its profile. We numerically solved the Boltzmann transport equations for each profile to determine the baryon asymmetry.

The effective potential approach we have employed has theoretical uncertainties that are diffi- 
cult to quantify without going through the arduous step of adding two-loop corrections. Ultimately a lattice study would be welcome for verifying the details of the phase transition properties, but this is an even more ambitious undertaking. ${ }^{15}$ A more tractable improvement on our treatment would be to rederive the transport equations and related coefficients, which we have taken from ref. [20] without modification. In particular, it is possible that including extra species in the network of equations could enhance the baryon asymmetry. Tau leptons, although weakly coupled, diffuse farther than other species, and could thus carry the CP asymmetry deeper into the symmetric phase and boost the integral (66) determining $n_{B} \cdot{ }^{16} \mathrm{~A}$ crucial issue to resolve concerns the correct form of the source term in the transport equations. Ref. [14] has recently claimed that 2HDMs can more readily produce a large enough baryon asymmetry (as well as address $B$ meson anomalies including the one we have considered) than we find. This is in part due to the use of a source term that is first order in derivatives, rather than the semiclassical force term with two derivatives that we have adopted.

Given these theoretical uncertainties in computing the baryon asymmetry, it seems reasonable to believe that the true prediction for a given model could differ by a factor of 2 from our determinations, despite the pains we have taken to get accurate results. At the same time, there is uncertainty in the value of the new Yukawa coupling parameter $\eta_{D}^{\prime}$ needed to match the $\mathrm{D} \varnothing$ anomaly; from table 17 of [4], we can estimate that the $1-\sigma$ error band in $h_{q}$ of eq. (16) is $h_{q}=0.258_{-0.80}^{+0.63}$ using the method advocated in [67]. Since $h_{q} \sim\left(\eta_{D}^{\prime}\right)^{2}$, this translates to the relative error $\delta \eta_{D}^{\prime} /\left|\eta_{D}^{\prime}\right|={ }_{-0.154}^{+0.122}$. From these considerations we can give a better picture of the tension between explaining the dimuon excess and baryogenesis as shown in fig. 13, It will be important to reduce the systematic errors in the prediction of the baryon asymmetry to know how serious the apparent tension really is.

One advantage of having a small allowed region of parameter space is that we can make somewhat distinctive predictions for collider searches. Typically the discovered mass spectrum is a light Higgs, and one or more heavy scalars below the $t \bar{t}$ mass threshold with branching ratios as shown in fig. 12, LHC can discover such a spectrum in the near future. This scenario for EWBG is falsifiable for most of the remaining allowed parameter space.

\footnotetext{
${ }^{15}$ Ref. [68] has also promoted a method to extract $v_{c} / T_{c}$ from the effective potential in a gauge-invariant manner.

16 We thank S. Tulin for pointing out this possibility
} 


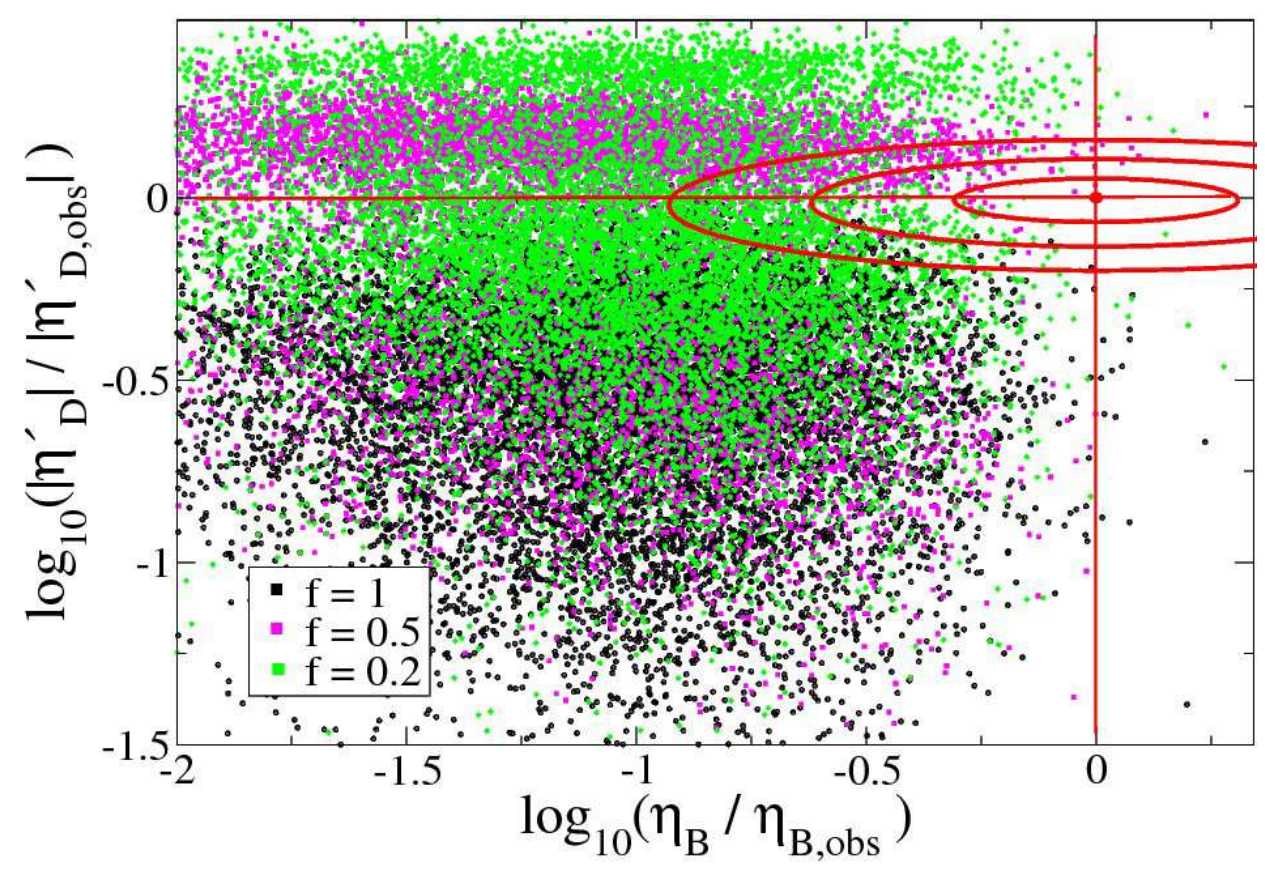

FIG. 13: Scatter plot of $\left|\eta_{D}^{\prime}\right|$ normalized to the observed value (as needed to explain the $\mathrm{D} \emptyset$ dimuon excess) versus the baryon asymmetry relative to its observed value. The 1,2 and 3- $\sigma$ error ellipses as described in the text are shown. Black points are for $f=1$, magenta for $f=0.5$, green for $f=0.2$, referring to $f$ defined in eq. (24).

\section{Acknowledgments}

JC thanks the particle theory group of the Perimeter Institute for its hospitality while this work was started, H. Logan and D. Neilsen for helpful correspondence or discussions, and the organizers and participants of "Electroweak Baryogenesis in the Era of the LHC" at the Weizmann Institute for stimulating discussions. MT thanks Mark Wise for discussions and collaboration related to material presented here and Pierre Savard for helpful communication. We thank Pietro Slavich for pointing out an error in the formula for $R_{b}$ in the original manuscript. 


\section{Appendix A: Counterterms}

Denoting derivatives of the Coleman-Weinberg potential $V_{\mathrm{CW}}$, evaluated at the tree-level VEVs, by $V_{1, x}$ and $V_{1, x y}$,

$$
\begin{aligned}
\delta \lambda v^{2} & =\frac{1}{2}\left(\frac{1}{v} V_{1, h}-V_{1, h h}\right) \\
\delta \mu^{2} & =\frac{1}{2}\left(\frac{3}{v} V_{1, h}-V_{1, h h}\right) \\
\delta \lambda_{3}^{R} v^{2} & =\frac{1}{2}\left(-V_{1, s_{R} s_{R}}+V_{1, s_{I} s_{I}}\right) \\
\delta \lambda_{3}^{I} v^{2} & =V_{1, s_{R} s_{I}} \\
\delta m_{1}^{2} & =-\frac{1}{2}\left(V_{1, s_{R} s_{R}}+V_{1, s_{I} s_{I}}\right) \\
\delta m_{2 R}^{2} & =\frac{1}{2}\left(V_{1, h s_{R}}-\frac{3}{v} V_{1, s_{R}}\right) \\
\delta m_{2 I}^{2} & =\frac{1}{2}\left(-V_{1, h s_{I}}+\frac{3}{v} V_{1, s_{I}}\right) \\
\delta \lambda_{5}^{R} v^{2} & =-V_{1, h s_{R}}+\frac{1}{v} V_{1, s_{R}} \\
\delta \lambda_{5}^{I} v^{2} & =-V_{1, h s_{I}}+\frac{1}{v} V_{1, s_{R}}
\end{aligned}
$$

As described in the text, the Goldstone boson contributions to $V_{C W}$ must be IR-regulated in order to give meaningful results.

\section{Appendix B: Field-dependent masses}

The neutral components of the complex scalar doublets can be written as

$$
H=\frac{1}{\sqrt{2}}\left(h+i h_{I}\right), \quad S=\frac{1}{\sqrt{2}}\left(s_{R}+i s_{I}\right)
$$

In our evaluation of the effective potential we are free to make a global $\mathrm{SU}(2)_{L}$ transformation that sets $h_{I}=0$ so that $V_{\text {eff }}$ depends only upon $h, s_{R}, s_{I}$. The resulting $4 \times 4$ neutral mass matrix, 
including finite- $T$ Debye mass corrections, has components given by

$$
\begin{aligned}
V_{h, h} & =\frac{\lambda}{4}\left(3 h^{2}-v^{2}\right)+\frac{1}{2}\left(\lambda_{1}+\lambda_{2}\right)\left(s_{R}^{2}+s_{I}^{2}\right)+\lambda_{3}\left(s_{R}^{2}-s_{I}^{2}\right)+3 h\left(\lambda_{5}^{R} s_{R}+\lambda_{5}^{R} s_{I}\right) \\
& +\left(3 \lambda+4 \lambda_{1}+2 \lambda_{2}+6 y_{t}^{2}+\frac{9}{2} g^{2}+\frac{3}{2} g^{\prime 2}\right) \frac{T^{2}}{24} \\
V_{h_{I}, h_{I}} & =\frac{\lambda}{4}\left(h^{2}-v^{2}\right)+\frac{1}{2}\left(\lambda_{1}+\lambda_{2}\right)\left(s_{R}^{2}+s_{I}^{2}\right)-\lambda_{3}\left(s_{R}^{2}-s_{I}^{2}\right)+h\left(\lambda_{5}^{R} s_{R}+\lambda_{5}^{R} s_{I}\right) \\
& +\left(3 \lambda+4 \lambda_{1}+2 \lambda_{2}+6 y_{t}^{2}+\frac{9}{2} g^{2}+\frac{3}{2} g^{2}\right) \frac{T^{2}}{24} \\
V_{s_{R}, s_{R}} & =m_{1}^{2}+\frac{1}{2}\left(\lambda_{1}+\lambda_{2}+2 \lambda_{3}\right) h^{2}+3 \lambda_{4}^{R} h s_{R}-\lambda_{4}^{I} h s_{I}+\lambda_{6}\left(3 s_{R}^{2}+s_{I}^{2}\right) \\
& +\left(4 \lambda_{1}+2 \lambda_{2}+12 \lambda_{6}+6\left|\eta_{t}\right|^{2}+\frac{9}{2} g^{2}+\frac{3}{2} g^{\prime 2}\right) \frac{T^{2}}{24} \\
V_{s_{I}, s_{I}} & =m_{1}^{2}+\frac{1}{2}\left(\lambda_{1}+\lambda_{2}-2 \lambda_{3}\right) h^{2}-3 \lambda_{4}^{I} h s_{I}+\lambda_{4}^{R} h s_{R}+\lambda_{6}\left(3 s_{I}^{2}+s_{R}^{2}\right) \\
& +\left(4 \lambda_{1}+2 \lambda_{2}+12 \lambda_{6}+6\left|\eta_{t}\right|^{2}+\frac{9}{2} g^{2}+\frac{3}{2} g^{2}\right) \frac{T^{2}}{24} \\
V_{h, h_{I}} & =\lambda_{3} s_{R} s_{I}+h\left(\lambda_{5}^{R} s_{I}-\lambda_{5}^{I} s_{R}\right) \\
V_{h, s_{R}} & =m_{2 R}^{2}+\left(\lambda_{1}+\lambda_{2}+2 \lambda_{3}\right) h s_{R}+\frac{1}{2} \lambda_{4}^{R}\left(3 s_{R}^{2}+s_{I}^{2}\right)-\lambda_{4}^{I} s_{R} s_{I}+\frac{3}{2} \lambda_{5}^{R} h^{2} \\
& +6\left(\lambda_{4}^{R}+\lambda_{5}^{R}+y_{t}\left|\eta_{t}\right| \cos \varphi_{\eta}\right) \frac{T^{2}}{24} \\
V_{h, s_{I}} & =-m_{2 I}^{2}+\left(\lambda_{1}+\lambda_{2}-2 \lambda_{3}\right) h s_{I}-\frac{1}{2} \lambda_{4}^{I}\left(s_{R}^{2}+3 s_{I}^{2}\right)+\lambda_{4}^{R} s_{R} s_{I}+\frac{3}{2} \lambda_{5}^{I} h^{2} \\
& +6\left(\lambda_{5}^{I}-\lambda_{4}^{I}-y_{t}\left|\eta_{t}\right| \sin \varphi_{\eta}\right) \frac{T^{2}}{24} \\
V_{s_{R}, s_{I}} & =h\left(\lambda_{4}^{R} s_{I}-\lambda_{4}^{I} s_{R}\right)+2 \lambda_{6} s_{I} s_{R} \\
V_{h_{I}, s_{R}} & =m_{2 I}^{2}+2 \lambda_{3} h s_{I}+\frac{1}{2} \lambda_{4}^{I}\left(3 s_{R}^{2}+s_{I}^{2}\right)+\lambda_{4}^{R} s_{R} s_{I}-\frac{1}{2} \lambda_{5}^{I} h^{2} \\
V_{h_{I}, s_{I}} & =m_{2 R}^{2}+2 \lambda_{3} h s_{R}+\frac{1}{2} \lambda_{4}^{R}\left(s_{R}^{2}+3 s_{I}^{2}\right)+\lambda_{4}^{I} s_{R} s_{I}+\frac{1}{2} \lambda_{5}^{R} h^{2} \\
& 6\left(\lambda_{4}^{R}+\lambda_{5}^{R}+y_{t}\left|\eta_{t}\right| \cos \varphi_{\eta}\right) \frac{T^{2}}{24} \\
& = \\
& = \\
& \\
& \\
&
\end{aligned}
$$

Here $\eta_{t} \equiv\left|\eta_{t}\right| e^{i \varphi_{\eta}}$ and we have denoted the real and imaginary parts of complex masses and couplings by $m_{2}^{2}=m_{2 R}^{2}+i m_{2 I}^{2}$, etc. The mass matrix must be numerically diagonalized to find the mass squared eigenvalues.

The charged scalar mass matrix elements are given by

$$
\begin{aligned}
V_{h^{+}, h^{-}} & =\frac{\lambda}{4}\left(h^{2}-v^{2}\right)+\frac{1}{2} \lambda_{1}\left(s_{R}^{2}+s_{I}^{2}\right)+h\left(\lambda_{5}^{R} s_{R}+\lambda_{5}^{I} s_{I}\right) \\
& +\left(3 \lambda+4 \lambda_{1}+2 \lambda_{2}+6 y_{t}^{2}+\frac{9}{2} g^{2}+\frac{3}{2} g^{\prime 2}\right) \frac{T^{2}}{24} \\
V_{h^{+}, s^{-}} & =\left(m_{2}^{2}\right)^{*}+\frac{1}{2} \lambda_{2} h\left(s_{R}+i s_{I}\right)+\lambda_{3} h\left(s_{R}-i s_{I}\right)+\frac{1}{2} \lambda_{4}^{*}\left(s_{R}^{2}+s_{I}^{2}\right)+\frac{1}{2} \lambda_{5} h^{2} \\
& +6\left(\lambda_{5}+\lambda_{4}^{*}+y_{t} \eta_{t}^{*}\right) \frac{T^{2}}{24}
\end{aligned}
$$




$$
\begin{aligned}
V_{s^{+}, h^{-}} & =V_{h^{+}, s^{-}}^{*} \\
V_{s^{+}, s^{-}} & =m_{1}^{2}+\frac{1}{2} \lambda_{1} h^{2}+h\left(\lambda_{4}^{R} s_{R}-\lambda_{4}^{I} s_{I}\right)+\lambda_{6}\left(s_{R}^{2}-s_{I}^{2}\right) \\
& +\left(4 \lambda_{1}+2 \lambda_{2}+12 \lambda_{6}+6\left|\eta_{t}\right|^{2}+\frac{9}{2} g^{2}+\frac{3}{2} g^{2}\right) \frac{T^{2}}{24}
\end{aligned}
$$

and the charged scalar mass eigenvalues are

$$
m_{ \pm}^{2}=\frac{1}{2}\left(V_{h^{+}, h^{-}}+V_{s^{+}, s^{-}}\right) \pm \frac{1}{2} \sqrt{\left(V_{h^{+}, h^{-}}-V_{s^{+}, s^{-}}\right)^{2}+4\left|V_{h^{+}, s^{-}}\right|^{2}} .
$$

The gauge boson masses in the basis of $W_{i}^{\alpha}, B^{\alpha}$ are given by

$$
\begin{aligned}
m_{W_{i}^{\alpha} W_{j}^{\beta}}^{2} & =\left(\frac{1}{4} g^{2} \phi^{2}+2 g^{2} T^{2} \delta_{\alpha, \|}\right) \delta_{i j} \delta_{\alpha \beta} \\
m_{W_{i}^{\alpha} B^{\beta}}^{2} & =\left(\frac{1}{4} g g^{\prime} \phi^{2}\right) \delta_{\alpha \beta} \\
m_{B^{\alpha} B^{\beta}}^{2} & =\left(\frac{1}{4} g^{\prime 2} \phi^{2}+2 g^{\prime 2} T^{2} \delta_{\alpha, \|}\right) \delta_{\alpha \beta}
\end{aligned}
$$

Here $\phi^{2}=h^{2}+s_{R}^{2}+s_{I}^{2}$ denotes the total electroweak-breaking VEV and $\delta_{\alpha, \|}$ indicates that only the longitudinal polarizations get a Debye correction at this order.

Finally, the top quark mass is given by

$$
m_{t}^{2}=\frac{1}{2}\left(\left(y_{t} h+\left|\eta_{t}\right|\left(s_{R} \cos \varphi_{\eta}-s_{I} \sin \varphi_{\eta}\right)\right)^{2}+\left|\eta_{t}\right|^{2}\left(s_{R} \sin \varphi_{\eta}+s_{I} \cos \varphi_{\eta}\right)^{2}\right)
$$

\section{Appendix C: Beta functions}

The scaled beta functions, generalizing the results of ref. [32], are as follows. We have defined $\lambda_{n}^{R, I}$ to be the real or imaginary part, respectively, of $\lambda_{n}$. By "scaled," we mean that $\beta_{i}=\hat{\beta}_{i} / 16 \pi^{2}$ in terms of the conventionally normalized function, $\beta_{\lambda}=d \lambda / d \ln \mu$, where $\mu$ is the renormalization scale.

$$
\begin{aligned}
\hat{\beta}_{\lambda}= & 6 \lambda^{2}+8 \lambda_{1}^{2}+8 \lambda_{1} \lambda_{2}+4 \lambda_{2}^{2}+16 \lambda_{3}^{2}+48\left|\lambda_{5}\right|^{2}+\frac{3}{2}\left(3 g^{4}+g^{\prime 4}+2 g^{2} g^{\prime 2}\right) \\
& -3 \lambda\left(3 g^{2}+g^{\prime 2}-4 y_{t}^{2}\right)-12 y_{t}^{4}, \\
\hat{\beta}_{\lambda_{1}}= & \left(\lambda+4 \lambda_{6}\right)\left(3 \lambda_{1}+\lambda_{2}\right)+4 \lambda_{1}^{2}+2 \lambda_{2}^{2}+8 \lambda_{3}^{2}+4\left|\lambda_{5}\right|^{2}+16\left(\lambda_{5}^{R} \lambda_{4}^{R}+\lambda_{5}^{I} \lambda_{4}^{I}\right)+4\left|\lambda_{4}\right|^{2}, \\
& +\frac{3}{4}\left(3 g^{4}+g^{\prime 4}-2 g^{2} g^{\prime 2}\right)-3 \lambda_{1}\left(3 g^{2}+g^{\prime 2}-2 y_{t}^{2}-2\left|\eta_{t}\right|^{2}-2\left|\eta_{b}\right|^{2}\right)-12 y_{t}^{2}\left|\eta_{t}\right|^{2}, \\
\hat{\beta}_{\lambda_{2}}= & \left(\lambda+4 \lambda_{6}\right) \lambda_{2}+8 \lambda_{1} \lambda_{2}+4 \lambda_{2}^{2}+32 \lambda_{3}^{2}+10\left|\lambda_{5}\right|^{2}+4\left(\lambda_{5}^{R} \lambda_{4}^{R}+\lambda_{5}^{I} \lambda_{4}^{I}\right)+10\left|\lambda_{4}\right|^{2}, \\
& +3 g^{2} g^{\prime 2}-3 \lambda_{2}\left(3 g^{2}+g^{\prime 2}-2 y_{t}^{2}-2\left|\eta_{t}\right|^{2}-2\left|\eta_{b}\right|^{2}\right)-12 y_{t}^{2}\left|\eta_{t}\right|^{2},
\end{aligned}
$$




$$
\begin{aligned}
\hat{\beta}_{\lambda_{3}}= & \left(\lambda+4 \lambda_{6}\right) \lambda_{3}+8 \lambda_{1} \lambda_{3}+12 \lambda_{2} \lambda_{3}+5\left|\lambda_{5}\right|^{2}+2\left(\lambda_{5}^{R} \lambda_{4}^{R}+\lambda_{5}^{I} \lambda_{4}^{I}\right)+5\left|\lambda_{4}\right|^{2}, \\
& -3 \lambda_{3}\left(3 g^{2}+g^{\prime 2}-2 y_{t}^{2}-2\left|\eta_{t}\right|^{2}-2\left|\eta_{b}\right|^{2}\right)-6 y_{t}^{2}\left|\eta_{t}\right|^{2}, \\
\hat{\beta}_{\lambda_{4}}^{R}= & 24 \lambda_{6} \lambda_{4}^{R}+6 \lambda_{1}\left(\lambda_{5}^{R}+\lambda_{4}^{R}\right)+4 \lambda_{2} \lambda_{5}^{R}+8 \lambda_{2} \lambda_{4}^{R}+4 \lambda_{3} \lambda_{5}^{R}+20 \lambda_{3} \lambda_{4}^{R} \\
& -3 \lambda_{4}^{R}\left(3 g^{2}+g^{\prime 2}-y_{t}^{2}-3\left|\eta_{t}\right|^{2}-3\left|\eta_{b}\right|^{2}\right)-12\left(\left(\eta_{t}^{R}\right)^{3}+\left(\eta_{t}^{I}\right)^{2}\left(\eta_{t}^{R}\right)\right) y_{t}, \\
\hat{\beta}_{\lambda_{4}}^{I}= & 24 \lambda_{6} \lambda_{4}^{I}+6 \lambda_{1}\left(-\lambda_{5}^{I}+\lambda_{4}^{I}\right)-4 \lambda_{2} \lambda_{5}^{I}+8 \lambda_{2} \lambda_{4}^{I}-4 \lambda_{3} \lambda_{5}^{I}-20 \lambda_{3} \lambda_{4}^{I} \\
& -3 \lambda_{4}^{I}\left(3 g^{2}+g^{\prime 2}-y_{t}^{2}-3\left|\eta_{t}\right|^{2}-3\left|\eta_{b}\right|^{2}\right)-12\left(\left(\eta_{t}^{I}\right)^{3}+\left(\eta_{t}^{R}\right)^{2}\left(\eta_{t}^{I}\right)\right) y_{t}, \\
\hat{\beta}_{\lambda_{5}}^{R}= & 6 \lambda \lambda_{5}^{R}+6 \lambda_{1}\left(\lambda_{5}^{R}+\lambda_{4}^{R}\right)+8 \lambda_{2} \lambda_{5}^{R}+4 \lambda_{2} \lambda_{4}^{R}+20 \lambda_{3} \lambda_{5}^{R}+4 \lambda_{3} \lambda_{4}^{R} \\
& -3 \lambda_{5}^{R}\left(3 g^{2}+g^{\prime 2}-3 y_{t}^{2}-\left|\eta_{t}\right|^{2}-\left|\eta_{b}\right|^{2}\right)-12 \eta_{t}^{R} y_{t}^{3}, \\
\hat{\beta}_{\lambda_{5}}^{I}= & 6 \lambda \lambda_{5}^{I}+6 \lambda_{1}\left(\lambda_{5}^{I}-\lambda_{4}^{I}\right)+8 \lambda_{2} \lambda_{5}^{I}-4 \lambda_{2} \lambda_{4}^{I}-20 \lambda_{3} \lambda_{5}^{I}+4 \lambda_{3} \lambda_{4}^{I} \\
& -3 \lambda_{5}^{I}\left(3 g^{2}+g^{\prime 2}-3 y_{t}^{2}-\left|\eta_{t}\right|^{2}-\left|\eta_{b}\right|^{2}\right)-12 \eta_{t}^{I} y_{t}^{3}, \\
\hat{\beta}_{\lambda_{6}}= & 24 \lambda_{6}^{2}+2 \lambda_{1}^{2}+2 \lambda_{1} \lambda_{2}+\lambda_{2}^{2}+4 \lambda_{3}^{2}+12 \lambda_{1}^{2}+\frac{3}{8}\left(3 g^{4}+g^{\prime 4}+2 g^{2} g^{\prime 2}\right) \\
& -3 \lambda_{6}\left(3 g^{2}+g^{\prime 2}-4\left|\eta_{t}\right|^{2}-4\left|\eta_{b}\right|^{2}\right)-6\left|\eta_{t}\right|^{4}-6\left|\eta_{b}\right|^{4} .
\end{aligned}
$$

[1] V. M. Abazov et al. [D0 Collaboration], arXiv:1106.6308 [hep-ex].

[2] V. M. Abazov et al. [D0 Collaboration], arXiv:1005.2757.

[3] V. M. Abazov et al. [ D0 Collaboration ], [arXiv:1109.3166 [hep-ex]].

[4] A. Lenz et al., arXiv:1008.1593.

[5] K. Ikado et al. [Belle Collaboration], Phys. Rev. Lett. 97, 251802 (2006) [arXiv:hep-ex/0604018].

[6] B. Aubert et al. [BABAR Collaboration], Phys. Rev. D 77, 011107 (2008) [arXiv:0708.2260 [hep-ex]].

[7] B. Aubert et al. [BABAR Collaboration], Phys. Rev. D 81, 051101 (2010) [arXiv:0809.4027[hep-ex]].

[8] I. Adachi et al. [Belle Collaboration], arXiv:0809.3834

[9] G. Blankenburg and G. Isidori, arXiv:1107.1216 [hep-ph].

[10] S. L. Glashow and S. Weinberg, Phys. Rev. D 15, 1958 (1977).

[11] R. S. Chivukula and H. Georgi, Phys. Lett. B 188 (1987) 99.

[12] L. J. Hall and L. Randall, Phys. Rev. Lett. 65, 2939 (1990).

[13] G. D’Ambrosio et al Nucl. Phys. B 645 (2002) 155 [arXiv:hep-ph/0207036].

[14] S. Tulin and P. Winslow, arXiv:1105.2848 [hep-ph].

[15] N. Turok and J. Zadrozny, Nucl. Phys. B 358, 471 (1991); Nucl. Phys. B 369, 729 (1992). 
[16] K. Funakubo, A. Kakuto and K. Takenaga, Prog. Theor. Phys. 91, 341 (1994) [arXiv:hep-ph/9310267].

[17] A. T. Davies, C. D. froggatt, G. Jenkins and R. G. Moorhouse, Phys. Lett. B 336, 464 (1994).

[18] J. M. Cline, K. Kainulainen and A. P. Vischer, Phys. Rev. D 54, 2451 (1996) [arXiv:hep-ph/9506284].

[19] M. Laine and K. Rummukainen, Nucl. Phys. B 597, 23 (2001) [arXiv:hep-lat/0009025].

[20] L. Fromme, S. J. Huber and M. Seniuch, JHEP 0611, 038 (2006) [arXiv:hep-ph/0605242].

[21] Z. Ligeti, M. Papucci, G. Perez and J. Zupan, arXiv:1006.0432] [hep-ph].

[22] K. Blum, Y. Hochberg and Y. Nir, arXiv:1007.1872,

[23] A. J. Buras, M. V. Carlucci, S. Gori and G. Isidori, arXiv:1005.5310 [hep-ph].

[24] A. J. Buras, G. Isidori and P. Paradisi, arXiv:1007.5291 [hep-ph].

[25] M. Trott and M. B. Wise, JHEP 1011, 157 (2010) [arXiv:1009.2813 [hep-ph]].

[26] T. Feldmann and T. Mannel, Phys. Rev. Lett. 100 (2008) 171601 [arXiv:0801.1802 [hep-ph]].

[27] A. L. Kagan, G. Perez, T. Volansky and J. Zupan, arXiv:0903.1794 [hep-ph].

[28] J. A. Bagger, K. T. Matchev and R. J. Zhang, Phys. Lett. B 412, 77 (1997) [arXiv:hep-ph/9707225].

[29] [LEP Higgs Working Group for Higgs boson searches.], arXiv:hep-ex/0107031.

[30] T. Aaltonen et al. [CDF Coll.], Phys. Rev. Lett. 103, 101803 (2009) [arXiv:0907.1269] [hep-ex]].

[31] V. M. Abazov et al. [D0 Collaboration], Phys. Rev. D 80, 051107 (2009) [arXiv:0906.5326 [hep-ex]].

[32] P. M. Ferreira and D. R. T. Jones, JHEP 0908, 069 (2009) [arXiv:0903.2856 [hep-ph]].

[33] I. F. Ginzburg and I. P. Ivanov, Phys. Rev. D 72, 115010 (2005) arXiv:hep-ph/0508020].

[34] D. A. Demir, M. Pospelov and A. Ritz, Phys. Rev. D 67, 015007 (2003) arXiv:hep-ph/0208257].

[35] G. Degrassi and P. Slavich, Phys. Rev. D 81, 075001 (2010) [arXiv:1002.1071 [hep-ph]].

[36] M. Jung, A. Pich and P. Tuzon, arXiv:1006.0470 [hep-ph].

[37] K. Nakamura et al. (Particle Data Group), J. Phys. G 37, 075021 (2010)

[38] B. Grzadkowski and M. Misiak, Phys. Rev. D 78, 077501 (2008) [arXiv:0802.1413 [hep-ph]].

[39] M. Ciuchini et al Nucl. Phys. B 527, 21 (1998) [arXiv:hep-ph/9710335].

[40] C. P. Burgess et al Phys. Lett. B 326, 276 (1994) arXiv:hep-ph/9307337].

[41] I. Maksymyk, C. P. Burgess and D. London, Phys. Rev. D 50, 529 (1994) [arXiv:hep-ph/9306267].

[42] C. P. Burgess, M. Trott and S. Zuberi, JHEP 0909, 082 (2009) [arXiv:0907.2696 [hep-ph]].

[43] J. M. Cline and P. A. Lemieux, Phys. Rev. D 55, 3873 (1997) arXiv:hep-ph/9609240.

[44] P. B. Arnold and O. Espinosa, Phys. Rev. D 47, 3546 (1993) [Erratum-ibid. D 50, 6662 (1994 PHRVA,D50,6662.1994)] [arXiv:hep-ph/9212235].

[45] R. R. Parwani, Phys. Rev. D 45, 4695 (1992) [Erratum-ibid. D 48, 5965 (1993 
PHRVA,D48,5965.1993)] [arXiv:hep-ph/9204216].

[46] S. J. Huber et al. Phys. Lett. B 475, 104 (2000) [arXiv:hep-ph/9912278].

[47] J. M. Cline, M. Joyce and K. Kainulainen, JHEP 0007, 018 (2000) [arXiv:hep-ph/0006119].

[48] L. Fromme and S. J. Huber, JHEP 0703, 049 (2007) [arXiv:hep-ph/0604159].

[49] M. Joyce, T. Prokopec and N. Turok, Phys. Rev. D 53, 2930 (1996) [arXiv:hep-ph/9410281]; Phys. Rev. D 53, 2958 (1996) [arXiv:hep-ph/9410282].

[50] J. M. Cline, M. Joyce, K. Kainulainen, Phys. Lett. B417, 79-86 (1998). [hep-ph/9708393]. J. M. Cline, K. Kainulainen, Phys. Rev. Lett. 85, 5519-5522 (2000). [hep-ph/0002272].

[51] K. Kainulainen, T. Prokopec, M. G. Schmidt, S. Weinstock, JHEP 0106, 031 (2001). [hep-ph/0105295]; Phys. Rev. D66, 043502 (2002). [hep-ph/0202177]. [hep-ph/0201293].

[52] T. Prokopec, M. G. Schmidt, S. Weinstock, Annals Phys. 314, 208-265 (2004). [hep-ph/0312110].

[53] T. Prokopec, M. G. Schmidt and S. Weinstock, Annals Phys. 314, 267 (2004) [arXiv:hep-ph/0406140].

[54] A. Riotto, Phys. Rev. D 53, 5834 (1996) [arXiv:hep-ph/9510271]; Nucl. Phys. B 518, 339 (1998) [arXiv:hep-ph/9712221].

[55] M. S. Carena et al. Nucl. Phys. B 503, 387 (1997) [arXiv:hep-ph/9702409].

[56] M. Herranen, K. Kainulainen, P. M. Rahkila, JHEP 1012, 072 (2010). arXiv:1006.1929 [hepph]]; J. Phys. Conf. Ser. 220, 012007 (2010). [arXiv:0912.2490 [hep-ph]]; JHEP 0905, 119 (2009). [arXiv:0812.4029 [hep-ph]]. Nucl. Phys. A820, 203C-206C (2009). [arXiv:0811.0936 [hepph]]; JHEP 0809, 032 (2008). [arXiv:0807.1435 [hep-ph]]; Nucl. Phys. B810, 389-426 (2009). [arXiv:0807.1415 [hep-ph]].

[57] V. Cirigliano, C. Lee, M. J. Ramsey-Musolf, S. Tulin, Phys. Rev. D81, 103503 (2010). [arXiv:0912.3523 [hep-ph]].

[58] V. Cirigliano, C. Lee, S. Tulin, [arXiv:1106.0747[hep-ph]].

[59] D0 Collaboration, D0 Note 6098-CONF

[60] CDF Collaboration, CDF Note 10206

[61] Y. Grossman, Y. Nir, G. Perez, Phys. Rev. Lett. 103, 071602 (2009). [arXiv:0904.0305][hep-ph]].

[62] Marta Calvi, for LHCb, FPCP 2011 Maale Hachamisha 23-27 May 2011 LHCb-CONF-2011-006

[63] A. Djouadi, Phys. Rept. 457, 1 (2008) [arXiv:hep-ph/0503172].

[64] S. Mantry, M. Trott, M. B. Wise, Phys. Rev. D77, 013006 (2008). [arXiv:0709.1505][hep-ph]].

[65] Atlas-Collaboration ATLAS-CONF-2011-087 http://cdsweb.cern.ch/record/1356196/files/ATLAS-CONF-2011-087.pd

[66] K. D. Lane and M. V. Ramana, Phys. Rev. D 44, 2678 (1991). 
[67] R. Barlow, [physics/0406120].

[68] H. H. Patel, M. J. Ramsey-Musolf, JHEP 1107, 029 (2011). [arXiv:1101.4665 [hep-ph]]. 